PALEO

Revue d'archéologie préhistorique

21 | 2009-2010

Varia

\title{
Le Vasconien : révision de sa signification à partir des industries lithiques d'Olha I et II, d'Isturitz et de Gatzarria
}

Marianne Deschamps

\section{OpenEdition}

Journals

Édition électronique

URL : http://journals.openedition.org/paleo/1775

DOI : $10.4000 /$ paleo. 1775

ISSN : 2101-0420

Éditeur

SAMRA

\section{Édition imprimée}

Date de publication : 1 mars 2011

Pagination : 103-126

ISSN : $1145-3370$

\section{Référence électronique}

Marianne Deschamps, «Le Vasconien : révision de sa signification à partir des industries lithiques d'Olha I et II, d'Isturitz et de Gatzarria », PALEO [En ligne], 21 | 2009-2010, mis en ligne le 21 octobre 2011, consulté le 07 juillet 2020. URL : http://journals.openedition.org/paleo/1775 ; DOI : https:// doi.org/10.4000/paleo. 1775

\section{c.) (i) (9)}

PALEO est mis à disposition selon les termes de la licence Creative Commons Attribution - Pas d'Utilisation Commerciale - Pas de Modification 4.0 International. 


\title{
LE VASCONIEN : révision de sa signification à partir des industries lithiques d'Olha I et II, d'Isturitz et de Gatzarria
}

\author{
Marianne DESCHAMPS ${ }^{(1)}$
}

\begin{abstract}
Résumé : En 1953, F. Bordes formalise la définition des faciès moustériens et en définit un nouveau, le Vasconien. Ce faciès se caractérise selon lui par la présence récurrente de hachereaux associés à un débitage Levallois et à des racloirs Quina dominants au sein de l'outillage. Le Vasconien a par la suite été remis en question par plusieurs auteurs qui ont mis en évidence une certaine hétérogénéité au sein de cet ensemble et ont proposé différentes interprétations. La question de la signification du Vasconien n'a pas été révisée depuis le développement des méthodes d'analyses liées à la technologie lithique. Nous présentons ici les études technologiques des industries d'Olha II (Cambo-les-Bains, Pyrénées-Atlantiques) et de Gatzarria (Ossas-Suhare, Pyrénées-Atlantiques). Les similitudes qu'elles révèlent et les comparaisons bibliographiques avec plusieurs séries des Monts cantabriques permettent de réévaluer le degré d'homogénéité des productions techniques anciennement attribuées au Vasconien sur des bases principalement typologiques. Une nouvelle interprétation de la signification de ce faciès au sein du Moustérien peut être proposée.
\end{abstract}

Mots-clés : Paléolithique moyen, Vasconien, Olha, Gatzarria, industrie lithique, hachereaux, Pyrénées-Atlantiques.

Key-words: Middle Palaeolithic, Vasconian, Olha, Gatzarria, lithic industry, Cleavers, Atlantic Pyrenees.

\begin{abstract}
Abridged english version
Vasconian: a revision of its signification based on Olha I and II, Isturitz and Gatzarria lithic industries.
\end{abstract}

"Vasconian" is a term proposed by F. Bordes to designate the Mousterian of the Vasco-cantabrian region. In this cultural definition that includes sites of Cantabric Mounts and Atlantic Pyrenees, the cleavers are unifying element of the associated industries. In the major part of the world, this tool has not been used since the end of Acheulean. The recurrent occurrence of cleavers that appears exclusively in this particular region for the Middle Palaeolithic raises numerous questions. F. Bordes thought that their production was related to an African influence. According to him, Vasconian is also characterized by a high percentage of scrappers and by the use of Levallois method.

Several authors questioned the existence of this facies, particularly L. G. Freeman (1966) who supports an economical interpretation to explain the reasons of changes in the composition of Mousterian implements. Later, V. Cabrera Valdes (1983) showed the heterogeneity of the typological and technical indices of the industries related to the Vasconian. From her works, the eventuality of coherent regional entity is discarded and the reason of the production of cleavers stays unresolved.

The history of the researches shows the problems of interpretation related to the Mousterian of this region. That is the reason why we decided to carry out a new evaluation of some of these series following a technological approach. We present here the first results of this work carried on in the framework of Ph.D. at Toulouse University. Does an homogeneity exist between the different series that were formerly attributed to the Vasconian mostly on typological grounds? 
Site's presentation

Olha II Rock Shelter (Cambo-les-Bains, Pyrénées-Atlantiques) is located near the stream called Olha, a few meters from its confluence with the Nive River (fig. 1). This site was discovered by G. Laplace in 1947 during his surveys close to Olha Rock Shelter. This location has been excavated at the beginnings of the twentieth century. The archaeological interest of this cavity was discovered in 1950. First excavations were directed by G. Laplace from 1956 until 1976. For these two collections, we carried out a sampling in order to favour a qualitative approach.

\section{Core reduction process}

At Olha II (fig. 8) and Gatzarria (fig. 9) sites, the Discoïd method is the most largely used. The blanks obtained by this core reduction process are mainly flakes with thick butts and peripheral cutting edges (fig. 10). The natural backed flakes and pseudo-Levallois points are also present but their lower percentage indicates that this group is not the main purpose of the core reduction process.

The second feature of the core reduction process shows unidirectional or bidirectional opposite platform modes. These cores are not numerous and can be considered as prismatic (fig. 11, fig. 13). This type has also been identified by J-M. Maillo Fernandez and his team through levels 11 and 12 of Morín Cave as well as through layer 20 of Castillo Cave (fig. 14, fig. 15). Other anecdotic production modes occur as multidirectional method or bipolar flaking.

Implement

Among tools identified in Olha II, scrappers make up the biggest group. The second biggest is composed by notches and denticulates. Points as well as tools of Upper Palaeolithic type are fewer. (fig. 17). Proportions of tool types are similar in the Cjr layer of Gatzarria, as we identify a majority of scrapers followed by notches and denticulates (fig. 16).

Implement economy: we have identified a high frequency of reshaping scars (fig. 17). This frequent reshaping in association with the high degree of core's exhaustion could be explained by the distance (about $10 \mathrm{~km}$ ) separating the knappers from the privileged outcrops. This topic will be more intensively studied in future works.

\section{Heavy Duty tools}

Handaxes: Twelve handaxes are identified in Olha I. Three morphologic types are recognized: almond shaped, transversal cutting edge, and canted handaxes (fig. 19) that seems to represent an intermediate type between the two latter. Morphologic differences can indicate functional differences.

Opening angle values of tips are always more elevated for canted handaxes than for almond shaped handaxes (fig. 20). The opening angles of the canted handaxes tips are all about $90^{\circ}$. That angle seems to be due to a morpho-technic rupture between the active part and one of the edges more than to a real active part.

Cleavers: Eighty-three cleavers are identified in Olha I. A specific study was conducted from this set as to understand the modalities and variabilities of the tool's production. Olha I cleavers majoritarily made on ophite, secondly on quartzite (fig. 21 , fig. 22). The chaîne opératoire of cleavers's production is not complete on the site, nor on any other site we studied. We suppose that production areas could be situated on supplying areas. The great majority of cleavers are of type 0 (fig. 24) according to Tixier's typology (1956). When the cutting edge has been predetermined, it seems that Olha I cleavers show a high degree of predetermination (fig. 25) as well as metrical standardization (fig. 26).

\section{Signification of cleavers production}

In Franco-cantabric region, Mousterian cleavers raise numerous questions, especially since their regular production is only present in that geographic area at that period. Several hypothesis are proposed to explain reasons for this particular production.

After selection, we can discard the hypothesis that this production is surviving since Acheulean times (Obermaier 1924 ; Vega del Sella 1921). Others hypothesis supporting environnemental or functional determinism are also to be discarded. As a result, we can consider that these tools constitute a cultural marker of a group in the Vasco-cantabric Mousterian (fig. 27).

\section{Discussion and further results}

We will conduct an experimentation to understand the reasons for the cleavers production. This type of approach has never been used on Mousterian cleavers. Various hypothesis of use patterns will be tested and cutting edges will be submitted to systematic use-wear analyses. The creation of a referential will allow comparison with the archaeological data.

An exhaustive technological study has to be made on Olha II and Gatzarria sites. We also planed to compare our results with layer 20 of Castillo Cave (Puente Viesgo, Spain), layer XIII of El Pendo Cave (Escobedo, Spain) as well as with layers 17 sup. 
to 13 at Morín Cave (Villanueva, Spain). This study will allow us to assess or discard the hypothesis of the existence of technical and cultural traditions from south Aquitaine through Cantabric Mounts.

Another part of this work deals with the chronology of this facies. Radiocarbon dates indicate that Vasconian about $40 \mathrm{Ka}$ old (OIS 3) (fig. 28), while faunal associations show temperate climate matching with the end of OIS 5 (Jaubert 2007). New archaeozoological investigations on Olha I and II assemblages will be carried out by M. C. Soulier (Toulouse University ph. D. student). Her work will precise the chronology of Vasconian and its environment.

Olha II and Gatzarria technological analyses put in light the unity of technological choices. The degree of this unity has to be tested by the study of several other sites. The use of technological methods to analyse lithic assemblages permits us to find back a real coherence in the cultural signification of Vasconian.

\section{Introduction}

Au sein du Paléolithique moyen, les productions techniques de l'homme de Néandertal présentent une diversité importante. Depuis les années 1930, des subdivisions à l'intérieur de ce grand ensemble ont été effectuées. D. Peyrony établit une première division en séparant les industries moustériennes à bifaces de celles sans bifaces (1930). F. Bordes et M. Bourgon précisent cette définition en 1951 en proposant le faciès du Moustérien de Tradition Acheuléenne. F. Bordes définira aussi quatre autres faciès qu'il caractérise par les types d'outils présents et leurs proportions. Dans son article de 1953 concernant la classification des industries moustériennes, il individualise aussi trois faciès régionaux qui n'entrent pas dans les cadres théoriques qu'il venait alors de poser : l'Asinipodien, le Pontinien et le Vasconien.

Il définit le Vasconien comme une entité regroupant des industries des Pyrénées-Atlantiques et des Monts cantabriques, et dont l'élément fédérateur est le hachereau. Largement répandu dans l'Acheuléen d'Afrique et d'Eurasie (Roche et Texier 1991 ; Texier 1996 ; Mourre 2003), cet outil n'est fréquent au Paléolithique moyen que dans la région vasco-cantabrique. C'est pourquoi sa présence soulève de nombreux questionnements. Outre cet outil, F. Bordes caractérise le Vasconien par la prépondérance des racloirs de type Quina et par un débitage Levallois. "Tout se passe comme si cette industrie avait reçu une infusion passagère de technique Levallois et de typologie africaine » (Bordes 1953 - p. 464).

La définition du Vasconien comme entité culturelle indépendante est rapidement remise en question par plusieurs auteurs qui proposent différentes interprétations, parfois contradictoires. Notamment L. G. Freeman (1966), en s'inspirant des travaux de S. et L. Binford (1966), proposera une explication fonctionnelle aux différences de composition des outillages moustériens. Les sites présentant des divergences au sein de l'outillage et de ses proportions sont interprétés par lui comme étant fonctionnellement complémentaires.

V. Cabrera Valdès s'opposera aussi à la validité du Vasconien (Cabrera Valdès 1983, 1984). La méthode d'analyse de F. Bordes lui permet de mettre en avant une grande hétérogénéité au sein des différentes séries qui ont été attribuées à ce faciès. Les indices typologiques et techniques présentant une grande variabilité, elle conclut que "L'apparition des hachereaux, même si elle est variable, doit être considérée comme un élément important, sans pour autant qu'elle serve de base pour créer un nouveau faciès, il faut simplement constater sa présence ${ }^{2}$ (Cabrera Valdès 1983 - p. 141).

Peu après, C. Chauchat reprend l'étude de l'industrie de l'abri Olha I qu'il attribue à une variante régionale du M.T.A. (Chauchat 1985). G. Laplace qui a interrompu la fouille de Olha Il quelques années plus tôt ne manquera pas de répondre afin d'exposer son désaccord avec la typologie empirique qui peut conduire à plusieurs interprétations : $F$. Bordes et C. Chauchat arrivent à des conclusions divergentes en utilisant pourtant la même méthode (Laplace 1986).

Plusieurs séries « vasconiennes » sont ainsi successivement attribuées à différents faciès moustériens par différents auteurs, et la question de l'existence d'une entité régionale reste finalement non résolue. L'idée restée prédominante est celle de V. Cabrera Valdès selon laquelle la diversité typologique et technique entre les ensembles est trop importante pour qu'ils forment une entité cohérente. Cependant depuis cette étude, il n'y a pas eu de nouveaux travaux cherchant à mettre en relation ces différentes séries par le biais de l'application des méthodes d'analyse de la technologie lithique.

L'historique des recherches $^{3}$ (Bordes 1953 ; Cabrera 1984 ; Chauchat 1985 ; Gonzalez Echegaray 1971, 1973 ; Freeman 1966 ; Laplace 1986 ; Laplace et Saenz de Buruaga 2000 ; Deschamps et Mourre sous presse) met en exergue les problèmes d'interprétation que pose le

(2) « La aparición de los hendedores, también variable, debe tenerse en cuenta como un elemento más, pero sin que sirvan de base para crear una facies nueva, sino simplemente haciendo constar su presencia. » (Cabrera Valdès 1983 - p. 141).

(3) Pour un historique des recherches détaillé du Vasconien voir Deschamps M. et Mourre V. (sous presse) - Le Vasconien, un demisiècle après sa définition par François Bordes, 134ème congrès national des sociétés historiques et scientifiques : "Célèbres ou obscurs, hommes et femmes, dans leur territoire et leur histoire ", colloque international François Bordes. 
Moustérien de cette région. C'est pourquoi nous avons choisi de réévaluer certaines de ces séries selon une approche technologique. Une homogénéité peut-elle être perçue entre les différentes séries anciennement attribuées au Vasconien sur des bases essentiellement typologiques ? Cette question permet aussi d'alimenter un débat plus vaste concernant les raisons et les implications de la diversité technique perceptible au Paléolithique moyen récent dans la partie occidentale de l'Europe.

L'étude technologique que nous avons mise en œuvre dans le cadre d'un mémoire de Master II (Deschamps 2008) et que nous poursuivons dans le cadre d'une thèse de doctorat à l'Université de Toulouse-le Mirail concerne les industries d'Olha I et II, de Gatzarria et d'Isturitz. Nous en exposons ici les premiers résultats. Pour chaque gisement, les industries des couches contenant des hachereaux ont été analysées sous forme de diagnostic technologique. Les sites d'Olha II et Gatzarria, fouillés par G. Laplace dans la seconde moitié du XXè siècle, présentent les séries les plus homogènes. L'industrie de la couche Askf-1 d'Olha II a donc fait l'objet d'une analyse plus complète afin d'être comparée aux ensembles de Gatzarria, Olha I et Isturitz.

\section{Présentation des sites}

L'abri effondré d'Olha II est découvert par G. Laplace à l'est de la commune de Cambo en 1947, à l'occasion de prospections effectuées à proximité de l'abri Olha. Les deux gisements sont situés dans le même massif calcaire à seulement quelques mètres l'un de l'autre (fig.1).

Le ruisseau Olha passe au pied du gisement et sa confluence avec la Nive se situe à quelques dizaines de mètres en aval du site. Un sondage est effectué de 1948 à 1952, suivi par une première période de fouille de 1953 à 1961. La lecture de la stratigraphie est complexe puisqu'un soutirage important est identifié. La seconde période de fouille de 1972 à 1977 permet de préciser la stratigraphie du site. L'abri Olha II a été fouillé selon la méthode "Laplace-Méroc » (Laplace et Méroc 1954) " par repérage de tous les objets à l'aide des coordonnées cartésiennes » (Laplace et Saenz de Buruaga 2000). Cet enregistrement systématique nous a permis d'analyser la répartition spatiale des vestiges lithiques par le biais de projections selon des coupes frontale et sagittale (fig. 2, fig. 3). Contrairement à ce qu'affirment les auteurs précédemment cités, seuls l'outillage et les nucléus ont été coordonnés. Nous avons pris en considération l'ensemble de la séquence afin de déterminer si des mélanges stratigraphiques étaient perceptibles. Certaines couches semblent en effet présenter des mélanges, notamment les deux plus hautes. En dessous, la couche Ask est la plus épaisse de la séquence stratigraphique ; elle est relativement pauvre en vestiges et ceux-ci semblent se concentrer plutôt à la base de la couche. L'épaisseur de cette couche (environ $80 \mathrm{~cm}$ ) est probablement liée à l'effondrement de l'abri, entièrement ou en partie, qui se caractérise par une ligne de blocs au cœur et au sommet de cette couche. En ce qui concerne la couche Askf-1, celle-ci s'individualise assez clairement ; elle ne présente pas de pendage, en coupe frontale comme en coupe sagittale et semble relativement homogène du point de vue de la dispersion des pièces dans l'épaisseur. À la base de la séquence, G. Laplace avait identifié des lentilles sableuses qui lui avaient permis de distinguer deux horizons (Slcgf1 et Slcgf2) au sein de la couche Slcg. La future étude archéozoologique menée par M.-C. Soulier pourra éventuellement apporter des précisions stratigraphiques complémentaires.

À l'heure actuelle, plusieurs questions subsistent concernant la stratigraphie d'Olha II et sa corrélation avec celle d'Olha I. II est vraisemblable que certains niveaux soient très proches technologiquement, cependant les indications de E. Passemard concernant la stratigraphie d'Olha I sont trop vagues pour déterminer si ces deux gisements correspondent ou non à un même site.

La Grotte de Gatzarria (Ossas-Suhare, PyrénéesAtlantiques) est située dans le Pays de Soule, au sein du massif des Arbailles, sur le versant nord-est du mont Hargagne. Si la grotte est connue depuis longtemps par les habitants de la région et les spéléologues, le potentiel archéologique de cette cavité ne fut découvert qu'en 1950 par P. Boucher et P. Bouillon. Dès 1951, des sondages furent effectués. En 1956, G. Laplace y réalisa les premières campagnes de fouille, interrompues en 1957 ; elles reprendront en 1961 jusqu'en 1976, toujours sous la direction de G. Laplace. Comme dans le gisement d'Olha II, la méthode appliquée à la fouille fut celle de "LaplaceMéroc » (1954).

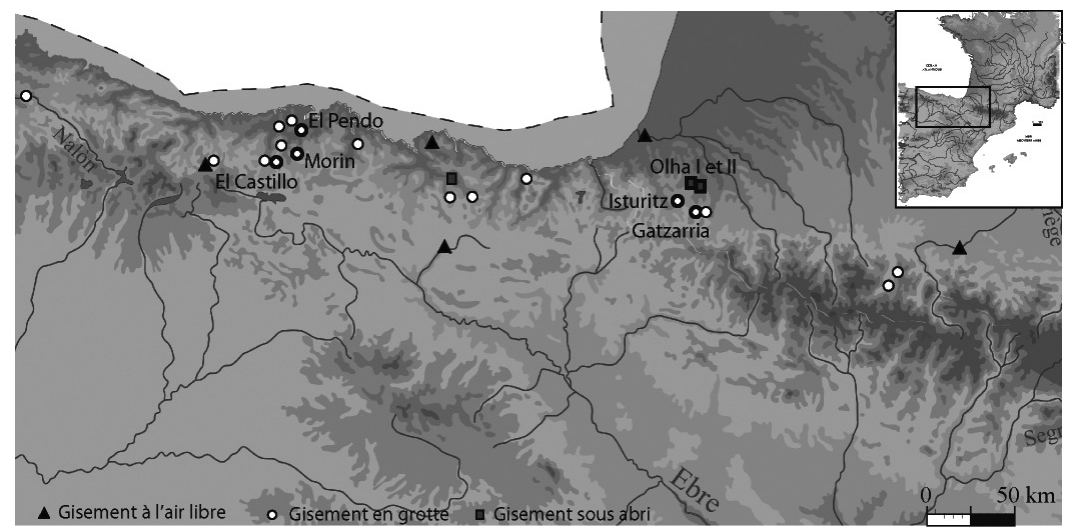

Figure 1 - Carte de répartition des principaux gisements moustériens cités dans le texte.

Figure 1 - Map of principal mousterian sites named in the text. 


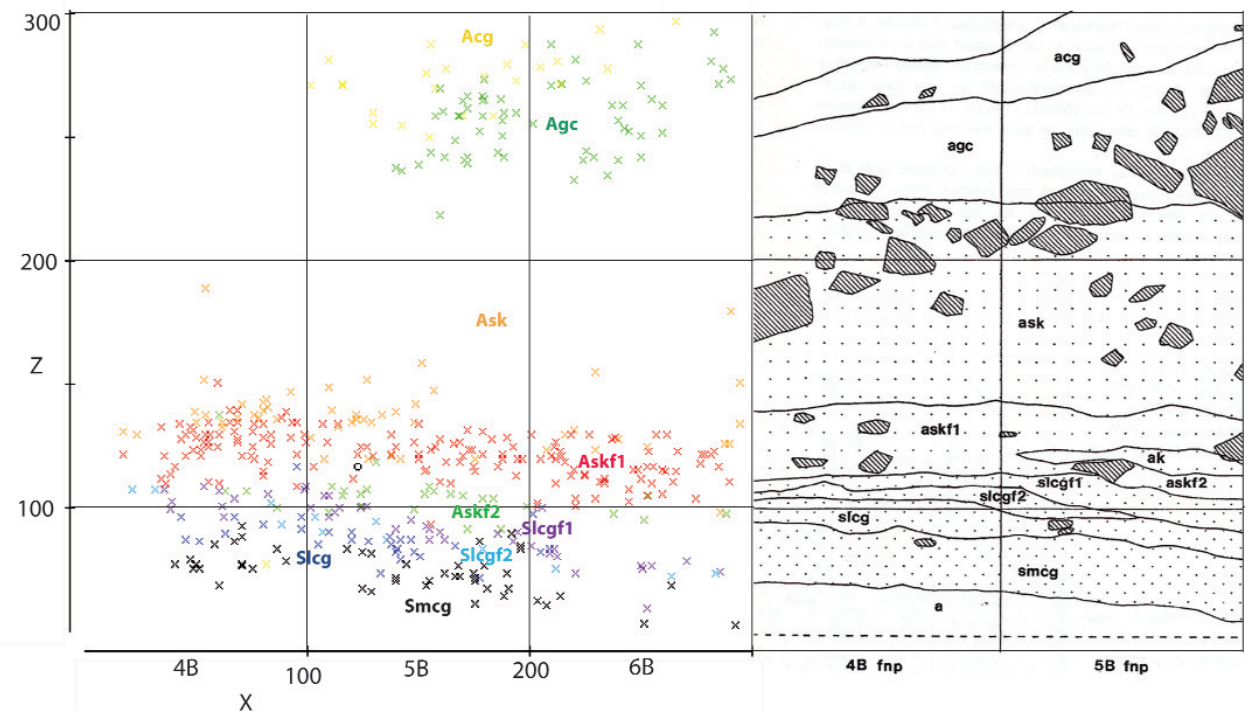

Figure 2 - Projection de la coupe frontale à partir des coordonnées des outils et des nucléus des carrés $4 B, 5 B$ et $6 B$ d'Olha ll et coupe frontale d'après Laplace et Saenz de Buruaga 2000.

Figure 2 - Projection of the frontal section from coordinated tools and cores from squares $4 B$, $5 B$ et $6 B$ of Olha II and frontal section after Laplace et Saenz de Buruaga 2000.

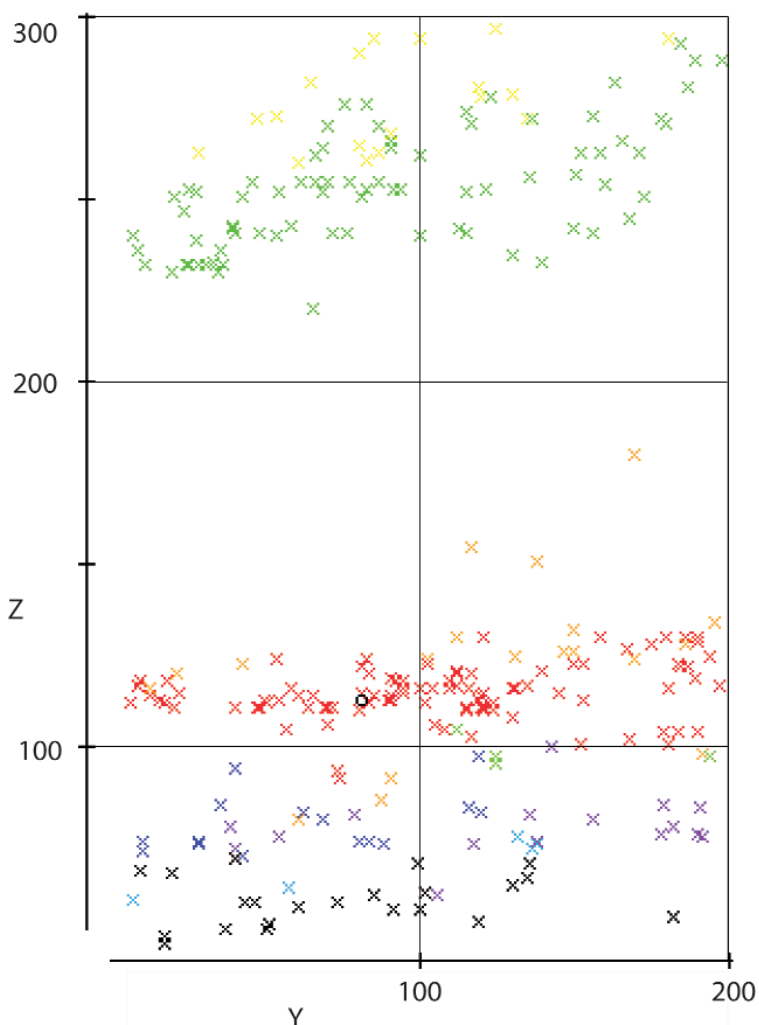

Figure 3 - Projection de la coupe sagittale d'Olha II à partir des coordonnées des outils et des nucléus des carrés $6 A$ et $6 B$.

Figure 3 - Projection of transversal section of Olha II from coordinated tools and cores from squares $6 A$ and $6 B$.

Dans une synthèse à propos des " complexes moustéroïdes de Gatzarria et d'Olha II " (Laplace et Saenz de Buruaga 2000), les auteurs proposent une étude des deux sites selon le principe de la typologie analytique ; ils cherchent à cerner les similitudes et les divergences entre les deux ensembles à partir d'analyses statistiques concernant les ordres et les groupes typologiques (Laplace 1957).
Ils concluent leur étude par la mise en évidence de liaisons morpho-techniques entre certains niveaux de Gatzarria et d'Olha II, sur la base desquelles ils définissent quatre faciès morpho-techniques qui présentent une dynamique évolutive diachronique (Laplace et Saenz de Buruaga 2002) : un faciès à denticulés plats (Olha II Sm) ; un faciès à pointes plates (Olha II SI) ; un faciès à hachereaux, divisé en deux sous-faciès : à pointes carénoïdes (Olha II As) et à racloirs carénoïdes (Gatzarria $\mathrm{Cjr}$ ) ; un faciès à racloirs plats et types leptolithiques (Gatzarria, Cj).

La grotte d'Isturitz forme le niveau supérieur d'un réseau karstique en trois étages qui s'est creusé au sein de la colline de Gatzelu, en Basse Navarre. Le potentiel archéologique de cette grotte est découvert par É. Piette lors d'exploitations de phosphate entre 1895 et 1898 . E. Passemard y mène les premières fouilles de 1912 à 1922. R. et S. de Saint-Périer y fouillent ensuite de 1928 à 1958 (Normand 2002). La grotte d'Isturitz fait actuellement l'objet de nouvelles fouilles sous la direction de C. Normand. Les travaux, repris en 2000 , concernent la salle de Saint-Martin.

E. Passemard (1944) établit une comparaison entre le niveau SV d'Isturitz et la couche Fi 3 de l'abri Olha car il fouille les deux gisements simultanément et perçoit des convergences entre ces industries, notamment à travers la présence d'un macro-outillage en ophite et quartzite. Lorsque $\mathrm{H}$. Delporte reprend l'étude de l'industrie d'Isturitz (Delporte 1974), il relativise la présence de hachereaux : «Il existe quelques grands objets qui tendent vers le hachereau sur éclat, mais aucun qui puisse être vraiment considéré comme typique » (Delporte 1974 - p. 29). Finalement, il considère que cette industrie présente des caractères atténués du Charentien, qui se confirmeront dans la couche M/SIV, sus-jacente. II approuve alors l'attribution de la couche P/SV au Vasconien de F. Bordes (1953). 


\section{Résultats préliminaires}

\section{Présentation des séries étudiées}

L'ensemble du matériel de la couche P/SV d'Isturitz a été étudié : la couche $\mathrm{P}$ (fouilles Passemard) a livré 91 pièces et la couche SV (fouilles Saint-Périer) 223 pièces. L'industrie de la couche Fi 3 d'Olha I compte 227 pièces.

Dans ces deux séries, le déficit des éclats bruts est important en comparaison avec le nombre d'outils présents. La collecte de la production lithique ne semble pas avoir été exhaustive puisque le nombre des éclats bruts est anormalement faible. En outre, dans l'outillage de la couche SV d'Isturitz, la présence de nombreuses pièces intrusives a été constatée (15,8\%), c'est donc la représentativité et l'homogénéité de cette série qui peuvent être remises en cause.

Ces ensembles présentent des biais trop importants pour pouvoir être intégrés à la reconstitution des chaînes opératoires. De même, les proportions de l'outillage ne sont pas fiables pour établir des comparaisons. Cependant, en ce qui concerne les hachereaux, l'étude de la série d'Olha I est pertinente puisqu'elle concerne des aspects qualitatifs liés à cet objet. Cette série constitue l'ensemble de hachereaux moustériens le plus important connu au nord des Pyrénées (83 pièces).

À Olha II, les fouilles ont été menées en deux temps. Nous avons choisi d'étudier le matériel provenant seulement de la seconde opération (1972-1977), d'une part parce que la zone de fouille des premières campagnes comportait un soutirage qui semble avoir perturbé les couches archéologiques et d'autre part, parce que le découpage stratigraphique établi lors des secondes fouilles paraît plus précis. Seul le matériel lithique de la couche Askf-1 a été étudié. Ce niveau a été fouillé sur une étendue de $7 \mathrm{~m}^{2}$; l'ensemble des nucléus et des outils a été étudié mais nous avons pris le parti d'échantillonner les produits de $2 \mathrm{~m}^{2}$ en ce qui concerne l'étude des produits bruts, afin de privilégier une approche qualitative de la série. Dans ce même souci, nous nous sommes référé parfois aux pièces provenant de la couche 2 (premières fouilles) lorsque celles-ci possédaient un potentiel informatif important. La richesse de cette couche a permis de bien évaluer la représentativité de la série. Notamment, au sein des produits bruts, de nombreuses pièces permettant une description détaillée des chaînes opératoires ont pu être identifiées et la fraction fine est bien représentée.

Les nucléus sont assez peu nombreux mais présentent des tendances communes qui permettent de décrire avec précision les principales chaînes opératoires de débitages attestées. L'analyse technologique montre que l'ensemble du débitage en silex, quartzite et quartz a été mené sur le site et la reconstitution des chaînes opératoires a pu être effectuée (fig. 4). II n'en est pas de même en ce qui concerne la production sur ophite puisque les outils (hachereaux) sont présents mais pas les nucléus ni les éclats de façonnage (un seul éclat d'ophite a été reconnu sur les deux carrés échantillonnés).

Concernant le matériel de la couche Cjr de Gatzarria, il semble que les carrés fouillés au fond de la grotte présentent des mélanges stratigraphiques. En effet, nous avons reconnu quelques pièces intrusives provenant probablement du

\begin{tabular}{|c|c|c|c|c|c|c|c|c|}
\hline & silex & quartzite & quartz & ophite & lydienne & grès & indéterminé & total \\
\hline outils & 410 & 27 & 9 & 7 & 0 & 1 & 2 & $\mathbf{4 5 6}$ \\
\hline nucléus & 27 & 3 & 5 & 0 & 0 & 0 & 0 & $\mathbf{3 5}$ \\
\hline $\begin{array}{c}\text { produits bruts } \\
\text { (5B et 6B) }\end{array}$ & 743 & 12 & 6 & 1 & 2 & 1 & 0 & $\mathbf{7 6 5}$ \\
\hline total & $\mathbf{1 1 8 0}$ & $\mathbf{4 2}$ & $\mathbf{2 0}$ & $\mathbf{8}$ & $\mathbf{2}$ & $\mathbf{2}$ & $\mathbf{2}$ & $\mathbf{1 2 5 6}$ \\
\hline
\end{tabular}

Figure 4 - Composition générale de l'industrie de la couche askf-1 d'Olha II.

Figure 4 - General composition of askf-1 layer's industry of Olha II.

\begin{tabular}{|c|c|c|c|c|c|c|c|c|}
\hline carré 3F & silex & quartzite & quartz & ophite & lydienne & grès & indéterminé & total \\
\hline outils & 57 & 93 & 3 & 0 & 1 & 0 & 0 & $\mathbf{1 5 4}$ \\
\hline nucléus & 1 & 13 & 0 & 0 & 1 & 0 & 0 & $\mathbf{1 5}$ \\
\hline $\begin{array}{c}\text { éclats bruts } \\
\text { de + de 2 cm }\end{array}$ & 11 & 1274 & 12 & 13 & 48 & 9 & 16 & $\mathbf{1 3 8 3}$ \\
\hline total & $\mathbf{6 9}$ & $\mathbf{1 3 8 0}$ & $\mathbf{1 5}$ & $\mathbf{1 3}$ & $\mathbf{5 0}$ & $\mathbf{9}$ & $\mathbf{1 6}$ & $\mathbf{1 5 5 2}$ \\
\hline
\end{tabular}

Figure 5 - Composition générale de l'industrie de la couche Cjr de Gatzarria.

Figure 5 - General composition of Cjr layer's industry of Gatzarria. 

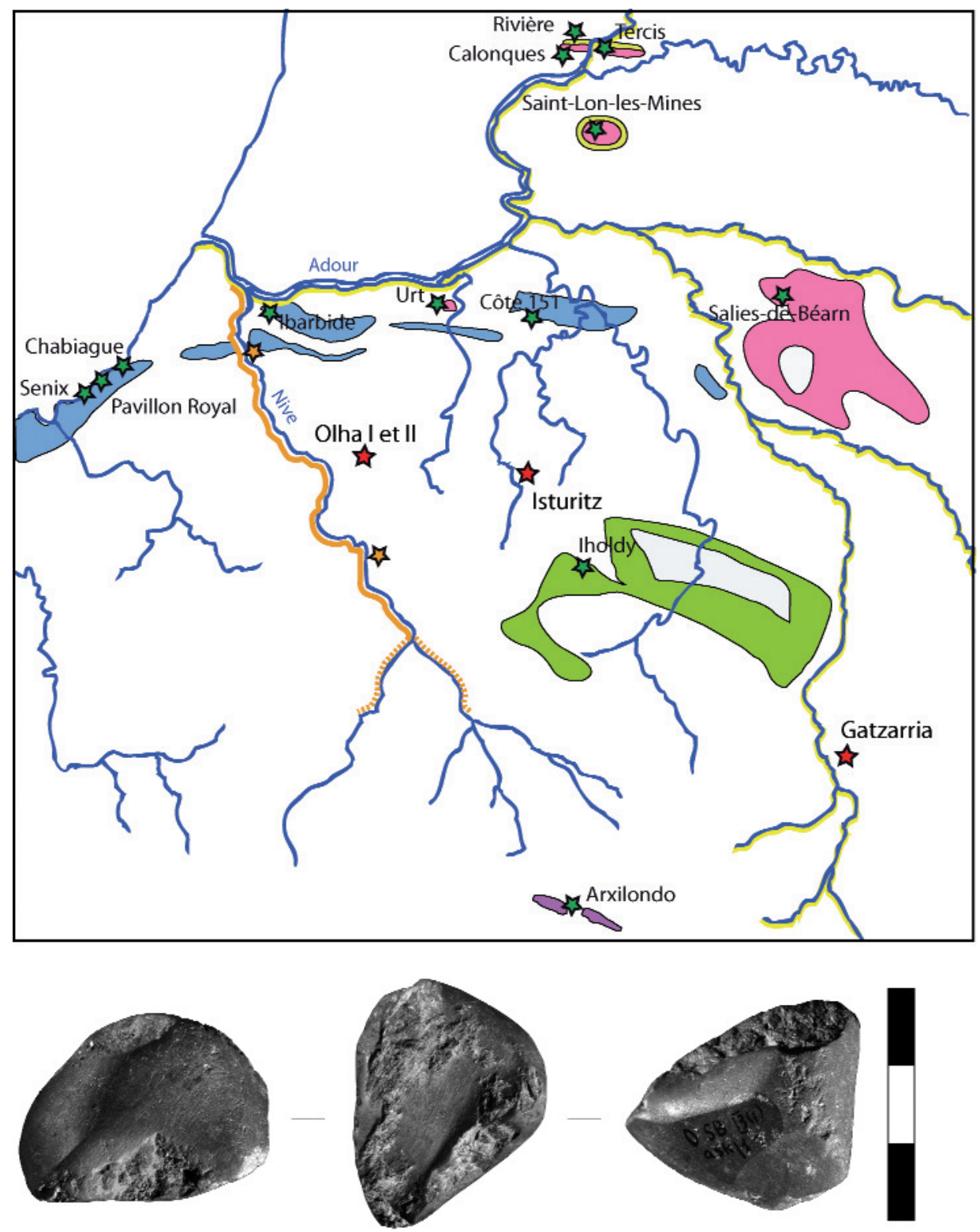

Figure 6 - Ressources lithiques à proximité des sites étudiés (d'après Normand 2002, modifié).

Figure 6 - Sources of raw material around the studied sites (after Normand 2002, modified). niveau proto-aurignacien, notamment au sein des outils. Nous avons donc orienté notre étude sur le matériel de l'entrée de la grotte ; face à la grande richesse de cette couche, nous avons limité l'étude des produits bruts et des outils à un échantillon correspondant au matériel du carré 3F (fig. 5).

\section{Matières premières}

L'approvisionnement en matière première est exclusivement local. À Olha II, I'utilisation du silex du Flysch domine largement ; il est disponible à environ une dizaine de kilomètres du site (fig. 6). Les affleurements de ce silex sont nombreux et constituent une ligne qui va jusqu'au littoral et se poursuit ensuite le long de la corniche cantabrique. Le silex identifié à Olha se présente sous la forme de plaquettes ou de rognons aplatis, ne dépassant pas 10 à $15 \mathrm{~cm}$.
Un quartzite fin a aussi été exploité sous forme de galets ne dépassant pas $10 \mathrm{~cm}$; sa provenance reste indéterminée. Des prospections dans les cours d'eau alentours vont être entreprises afin de la déterminer plus précisément. Enfin, l'introduction de l'ophite sur l'ensemble de ces sites semble résulter d'un approvisionnement particulier lié à la production des hachereaux. Cette matière est présente à proximité du site, sous la forme de galets entre 10 et $30 \mathrm{~cm}$. Des galets de modules plus importants sont disponibles dans la Nive, en amont. Seuls les produits finis sont cependant importés sur les sites. Une autre matière première lithique a été exploitée, dont la rareté en contexte moustérien implique qu'on la mentionne : plusieurs fragments d'hématites ont été identifiées à Olha II, dont un qui présente des facettes de polissage (fig. 7).

Le site de Gatzarria est plus proche de la chaîne axiale des Pyrénées. Si le quartzite est majoritaire, la lydienne et le 
quartz hyalin sont aussi présents. Du silex d'Iholdy, gîte le plus proche, est également présent. On peut donc considérer que le déterminisme de la matière première est faible et qu'il n'intervient pas sur les types de productions effectuées puisque nous allons voir qu'au sein des modes opératoires de production d'éclats, des similitudes sont observables entre les deux séries.

\section{Chaînes opératoires de production de supports}

Le débitage Discoïde (Mourre 2003) domine à Olha II (fig 8) comme à Gatzarria (fig. 9). Sur l'ensemble des séries étudiées, une hiérarchisation entre les surfaces est fréquemment identifiée notamment par le biais de zones corticales résiduelles majoritaires sur une des deux surfaces ; elles sont assez planes et l'axe de débitage est majoritairement centripète.

Bien que les supports sur lesquels s'effectue le débitage n'ont fréquemment pas pu être identifiés, la présence d'éclats de type Kombewa en quantité restreinte $(2,1 \%$ des produits bruts) indique que le débitage débute parfois sur face inférieure d'éclat. L'aménagement des surfaces de plan de frappe est représenté par la présence d'éclats plus larges que longs et présentant une zone distale corticale. Ces éclats possèdent de plus une légère courbure distale. Les nucléus en quartzite ne présentent en général pas d'aménagement du plan de frappe, la surface néo-corticale servant le plus souvent telle quelle.

Les supports issus de ce débitage sont majoritairement des éclats à talon épais et à tranchant périphérique. Les éclats à dos débordants et les pointes pseudo-Levallois sont aussi présents mais leur nombre plus restreint indique qu'ils ne constituent pas l'objectif principal du débitage (fig. 10). La faible représentation des axes de débitage cordaux le confirme. Puisque les surfaces de débitage sont relativement planes, les éclats débordants ont certainement un rôle au sein d'une phase de remise en forme des convexités, afin d'abaisser ou de rééquilibrer le plan d'intersection entre ces surfaces.

La présence d'éclats à crête axiale ou transversale est de même liée à une phase de remise en forme des convexités. Le détachement d'un éclat à crête permet de rehausser le plan d'intersection des surfaces (Slimak 2004). Cette nouvelle surface va ensuite servir " [...] comme plan de frappe préférentiel à des séquences unipolaires permettant à la fois la poursuite du débitage et une diminution de la convexité du nucléus 》(Slimak 2004 - t. 1, p. 70). L'ouverture d'une troisième surface peut aussi permettre un passage vers un débitage polyédrique ou marquer simplement la fin du débitage. La présence de ces éclats laisse supposer que la convexité de la surface de débitage a dû être plus importante durant certaines phases. Les surfaces planes observées semblent donc bien être le reflet du degré élevé d'exhaustion des nucléus.

Un second mode de débitage consiste en une production unipolaire ou bipolaire sur une surface assez cintrée. Ces nucléus, même s'ils sont peu nombreux, sont présents à
Olha II $(n=5)$ comme à Gatzarria $(n=9)$, et peuvent être qualifiés de nucléus prismatiques (fig. 11, fig. 13). Les flancs adjacents à la surface de débitage sont en continuité avec celle-ci. Le plan de frappe est toujours lisse, parfois sur un pan de fracture naturelle et est disposé à l'orthogonal de la surface de débitage. La surface de débitage, de forme rectangulaire ou triangulaire, possède des convexités latérales assez marquées alors que les convexités distales le sont peu. Comme dans le cas des nucléus discoïdes, les dimensions sont assez réduites, il est cependant délicat d'évaluer le degré d'exhaustion de cette production puisque la présence résiduelle de cortex sur les flancs de certaines pièces semble indiquer une séquence de débitage assez courte. Les phases de mise en forme semblent assez sommaires, des éclats sont directement détachés en direction de la surface de débitage depuis un plan de frappe parfois naturel ce qui suppose une sélection des supports selon des caractéristiques morphologiques et volumétriques.

Le degré d'exhaustion rend difficile la lecture de l'enchaînement opératoire et les produits issus de ce débitage prismatique sont complexes à discriminer. D'après les négatifs présents sur les nucléus, les produits recherchés sont de petits supports allongés à bords parallèles. Une telle composante a en effet été reconnue au sein des produits bruts mais le manque de caractéristiques communes les regroupant pose des problèmes d'identification (fig. 12).

Ce type de nucléus a aussi été identifié par J-M. Maillo Fernandez et son équipe dans les couches 20 et 21 de la grotte du Castillo (fig. 14, fig. 15) ainsi que dans les couches 11 et 12 de la grotte Morin (Maillo Fernandez et al. 2004, 2007). Ces nucléus présents sur plusieurs sites de Cantabrie évoquent, selon les auteurs, une production lamellaire. Concernant cette production à Olha II et Gatzarria, si la conception du nucléus peut effectivement être qualifiée de prismatique, les produits issus de ce débitage restent difficilement reconnaissables. De plus, ces produits ont rarement servi de supports d'outils, ce qui complexifie encore leur identification.

D'autres modes de production d'éclats sont présents de manière plus anecdotique, comme le débitage sur enclume, représenté par quelques rares pièces à Gatzarria (1,5\% des nucléus). Ici, c'est un vrai débitage d'éclat qui est mené selon cette méthode, alors que le rôle de la percussion sur enclume à Olha II se restreint à l'ouverture de galets.

Les chaînes opératoires de production d'éclats sur les sites de Olha II et de Gatzarria présentent donc des similitudes importantes (débitage Discoïde à surfaces hiérarchisées majoritaire ; débitage prismatique), bien que les matières premières impliquent quelques différences, notamment en ce qui concerne le gabarit général de la production. Les séries d'Olha I et d'Isturitz, malgré le problème de leur représentativité, possèdent les mêmes caractéristiques en ce qui concerne le débitage ; le concept Discoïde y est majoritaire (88\% à Olha I et $65 \%$ à Isturitz). Cependant, le débitage prismatique reconnu à Olha II et Gatzarria n'a pas, ici, été identifié. Des similitudes peuvent aussi être établies à une plus grande échelle, avec plusieurs sites des Monts cantabriques. 

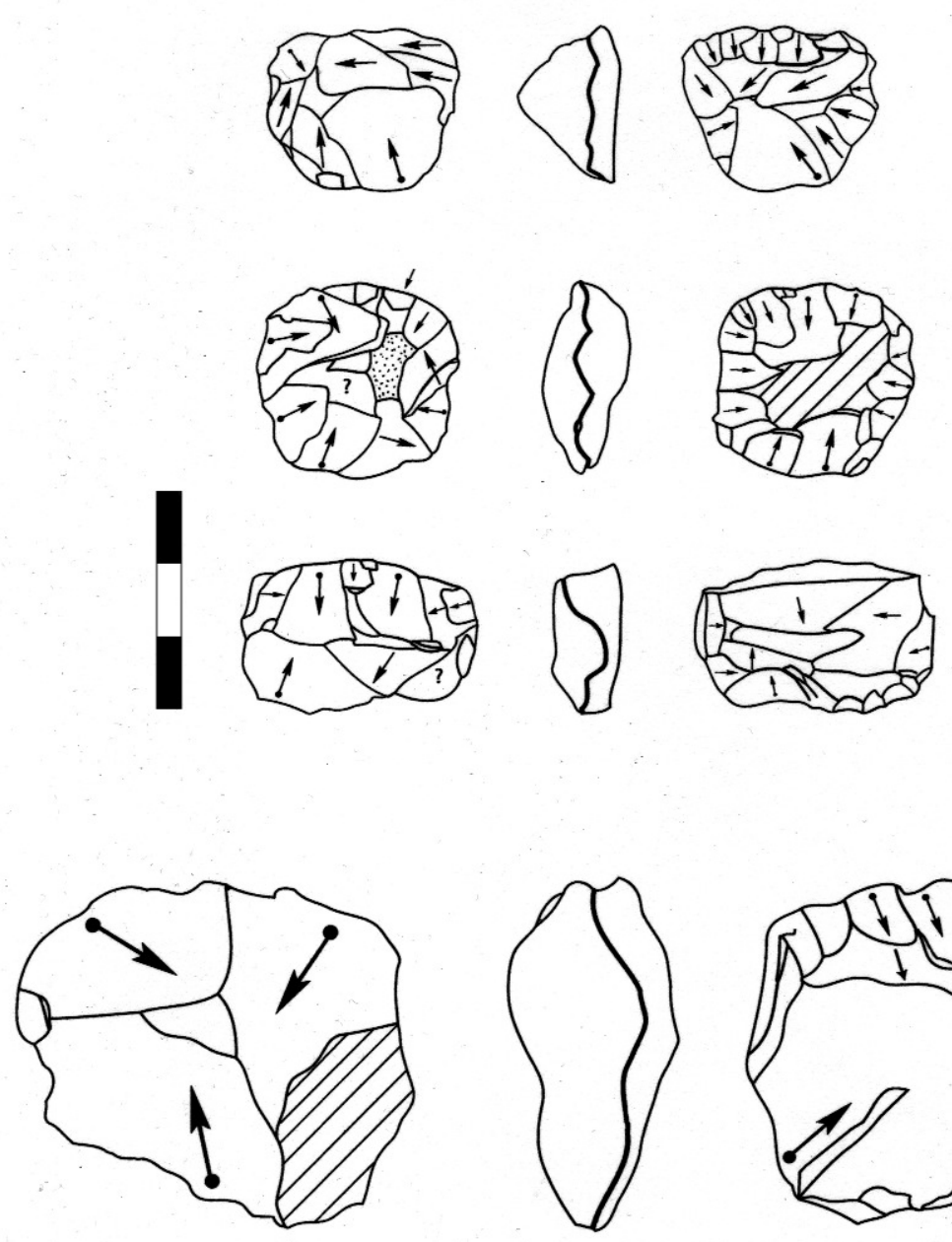

VIA Face inférieure d'éclat Néo-cortex

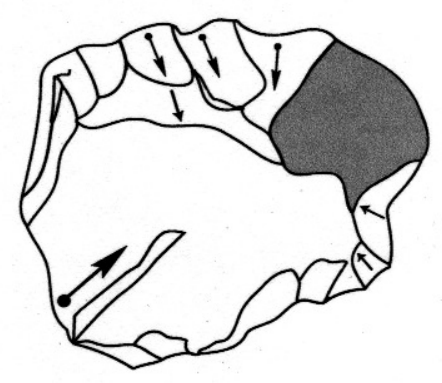

Figure 8 - Schémas diacritiques de nucléus discoïdes d'Olha II. Figure 8 - Schematic representation of discoïdes cores at Olha II.
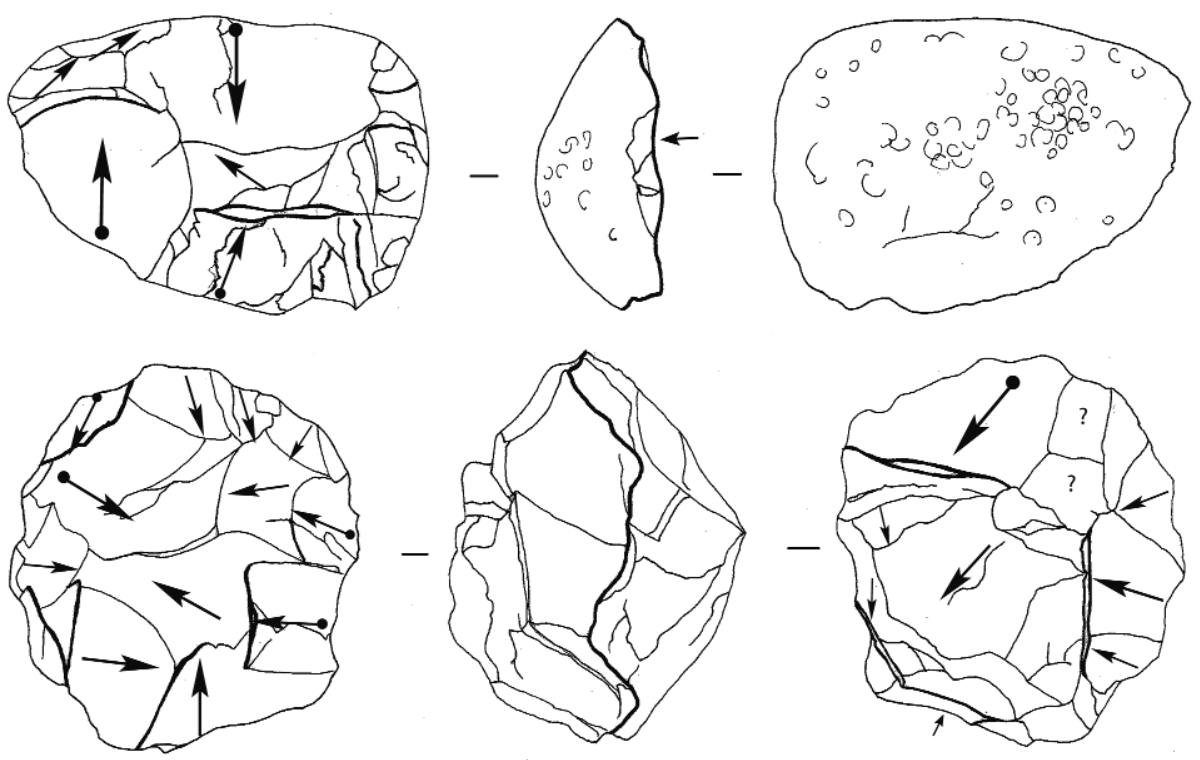

Figure 9 - Schémas diacritiques de nucléus discoïdes de Gatzarria.

Figure 9 - Schematic representation of discoïdes cores at Gatzarria. 

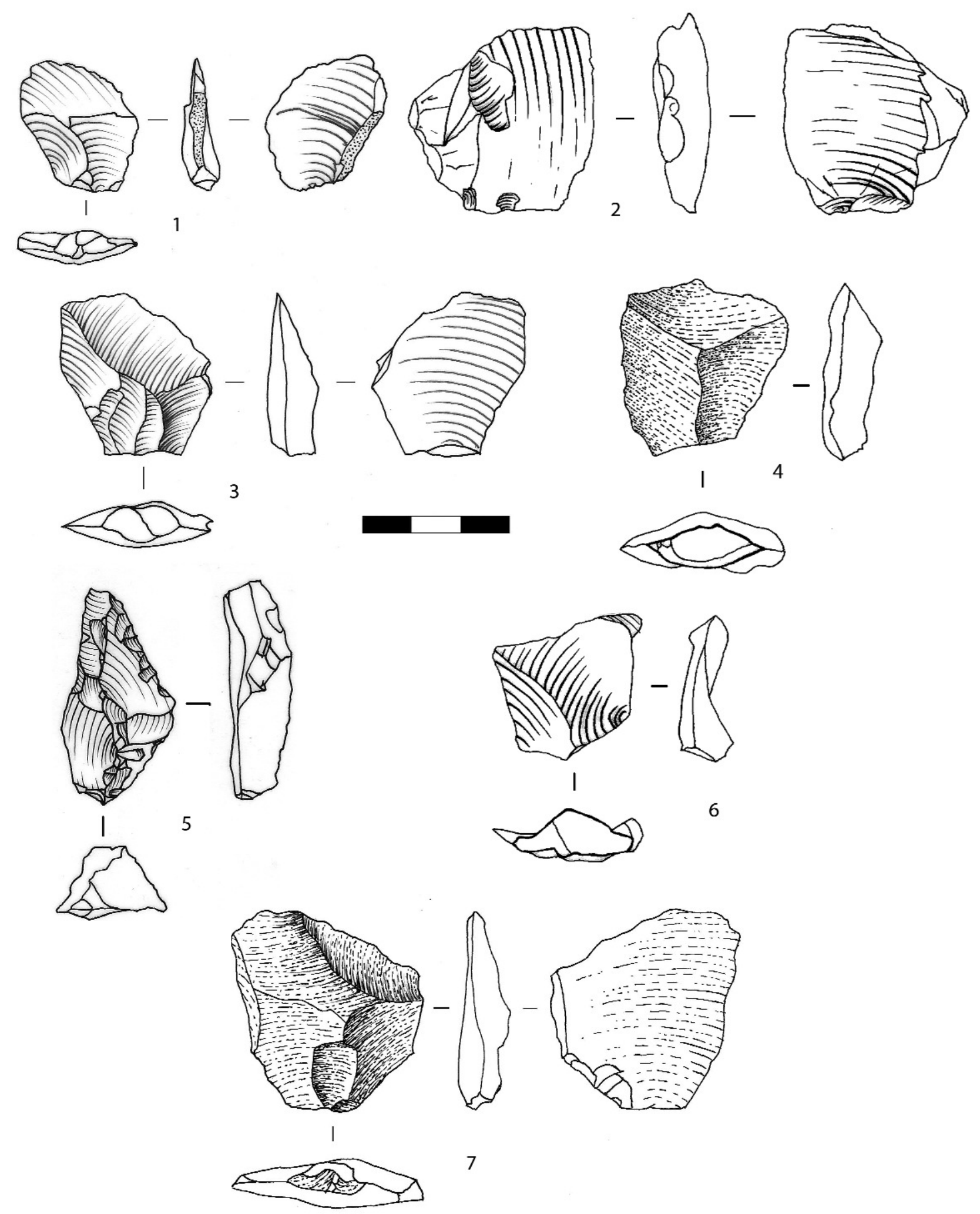

Figure 10 - Éclats bruts provenant du débitage Discoïde. 1 : éclat débordant ; 2 : éclat Kombewa ; 3 et 4 : éclats à tranchants périphériques ; 5 : éclat à crête axiale ; 6 et 7 : pointes pseudo-Levallois.

Figure 10 - Unretouched flakes obtained by Discoïdal core reduction. 1 : backed flake ; 2 :Kombewa flake ; 3 and 4 : flakes with peripherical cutting edge ; 5 : axial crested flake ; 6 et 7 : pseudo-Levallois points. 

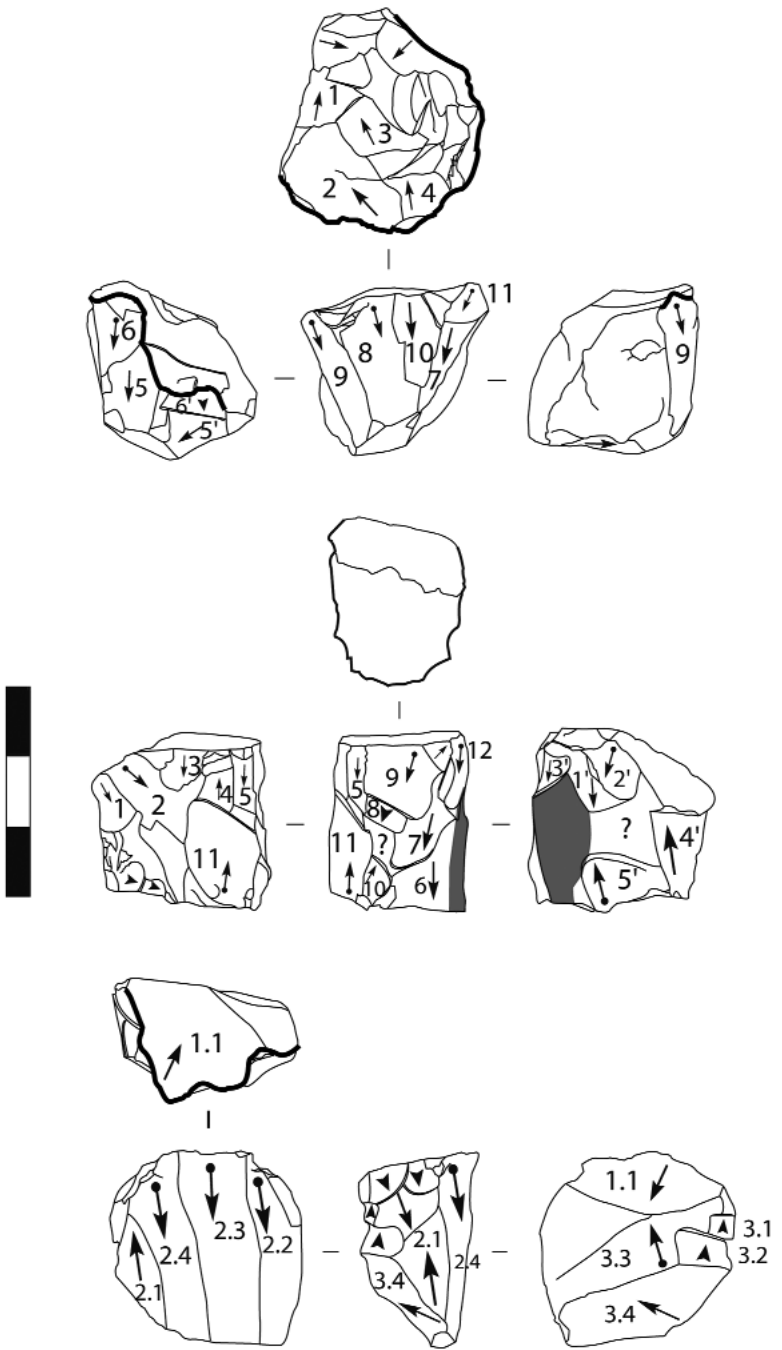

Figure 11 - Nucléus prismatiques d'Olha II.

Figure 11 - Prismatic cores at Olha II.
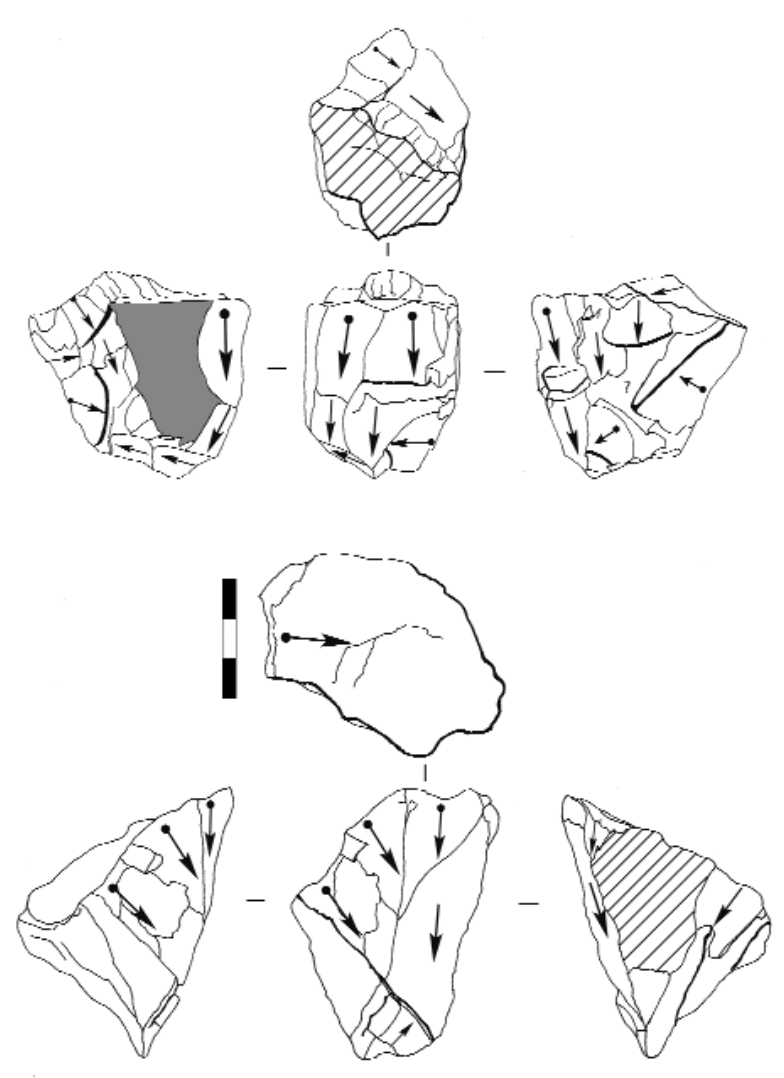

Figure 13 - Nucléus prismatiques de Gatzarria.

Figure 13 - Prismatic cores at Gatzarria.
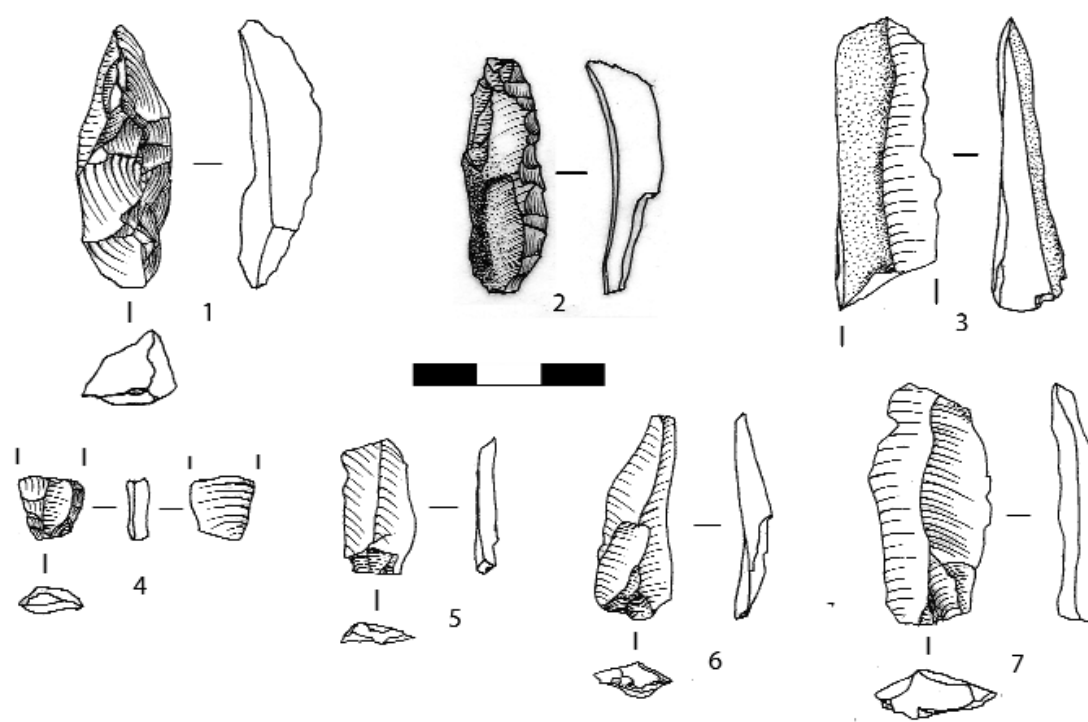

Figure 12 - Produits issus du débitage prismatique. 1 : crête ; 2 : éclat d'entame retouché ; 3 : lame corticale ; 4 : fragment proximal, d'éclat retouché : 5 à 7 : éclats allongés.

Figure 12 - Flakes obtained by prismatic core reduction. 1 : crest ; 2 : retouched first flake ; 3 : cortical blade ; 4 : proximal fragment of retouched flake; 5 to 7 : elongated flakes. 


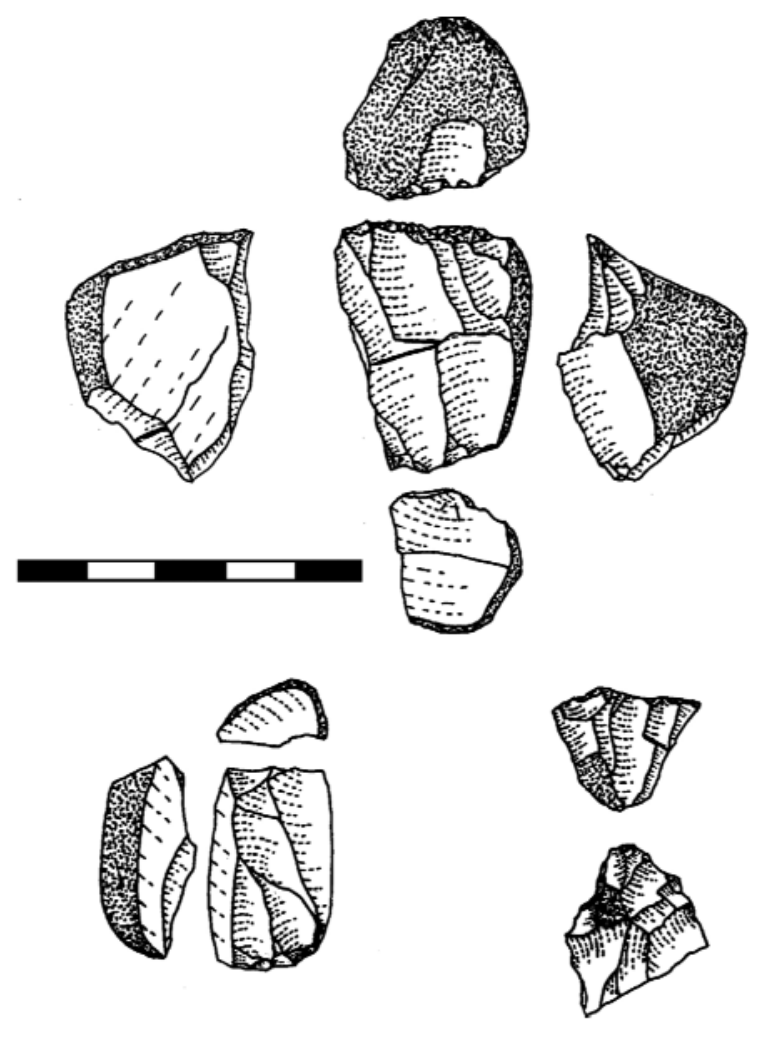

Figure 14 - Nucléus provenant du niveau 21 de la grotte du Castillo (Maílo Fernandez et al. 2004).

Figure 14 - Cores from layers 21 at Castillo Cave(Maílo Fernandez et al. 2004).

\section{L'outillage : produits retouchés}

\section{Typologie et proportions}

Au sein de l'outillage identifié à Olha II, les racloirs, majoritairement simples latéraux, constituent le groupe dominant. Le second groupe, le plus important, est celui des encoches et denticulés. Les pointes ainsi que les outils de type Paléolithique supérieur sont par contre peu représentés.

Dans la couche Cjr de Gatzarria, les proportions des types d'outils sont similaires à celles d'Olha II puisqu'on identifie une majorité de racloirs suivis du groupe des encoches et denticulés tandis que les pointes restent rares (fig. 16).

Dans sa définition du Vasconien, F. Bordes parle des racloirs de type Quina comme l'une des caractéristiques de ce faciès. Nous n'avons pas reconnu, à Olha II comme à Gatzarria, de nucléus Quina (Bourguignon 1997) ou de support d'outils épais renseignant ce débitage, pas plus qu'une proportion d'outils remarquable portant une retouche scalariforme de type Quina (3,4 \% de l'outillage). Concernant les racloirs (fig $17, \mathrm{n}^{\circ} 1,2$ ), la retouche est majoritairement directe et écailleuse, d'inclinaison semiabrupte. Son étendue est le plus souvent courte et sa délinéation est convexe ou rectiligne dans la majorité des cas.

Les denticulés (fig. 17, $n^{\circ} 5,7$ ) sont confectionnés par une série d'encoches clactoniennes adjacentes. Nous avons

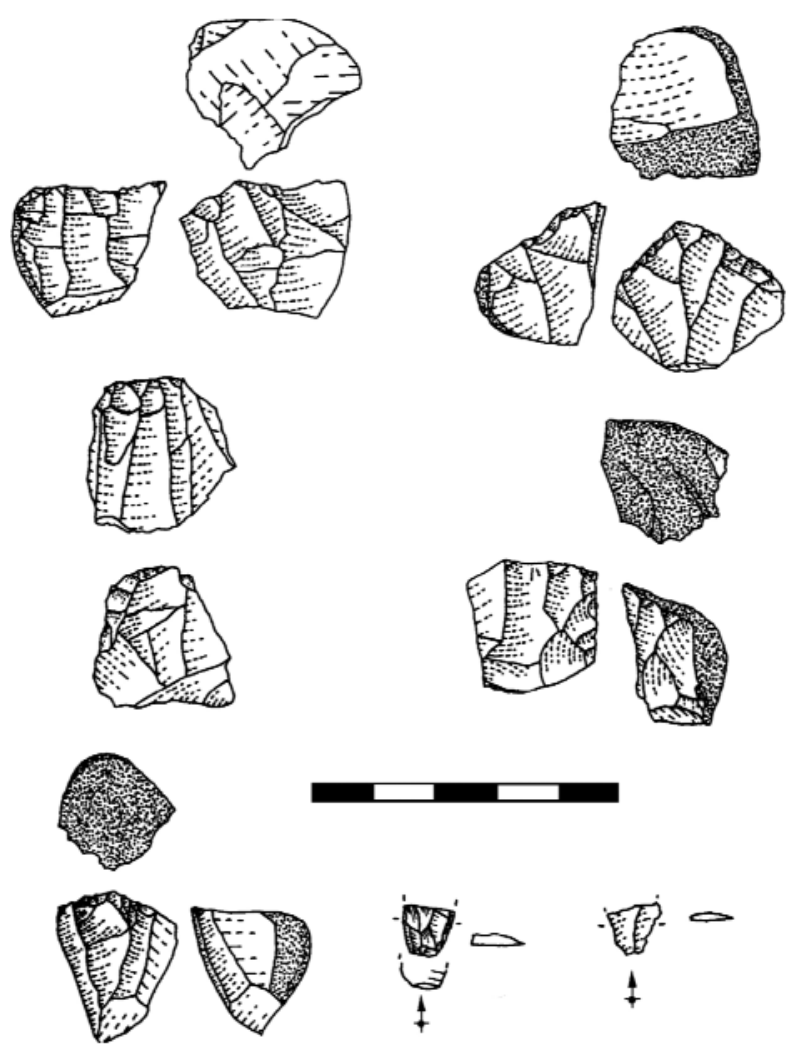

Figure 15 - Nucléus provenant du niveau 20 c et 20 ab de la grotte du Castillo (Maíllo Fernandez et al. 2004).

Figure 15 - Cores and flakes from layers $20 \mathrm{c}$ and $20 \mathrm{ab}$ at Castillo Cave (Maíllo Fernandez et al. 2004).

essayé de rester prudent en ce qui concerne le caractère anthropique de ces retouches puisque comme $\mathrm{C}$. Thiébaut l'a démontré (2005), de nombreux facteurs taphonomiques peuvent être la cause d'impacts donnant à la pièce une morphologie denticulée. Toute pièce douteuse a donc été écartée. La position de la retouche est majoritairement directe mais une retouche inverse a été plus fréquemment pratiquée sur les racloirs.

Les encoches sont majoritairement réalisées par des encoches clactoniennes ; elles ont donc une tendance à être assez profondes.

Des éléments appointés (fig. 17, $n^{\circ} 4,6,8,9$ ) sont confectionnés sur divers types de supports (pointes, pointes pseudo-Levallois, éclats de façonnage, ou éclat épais), la retouche est majoritairement directe, écailleuse ou subparallèle.

Des éléments triangulaires lors du débitage ont été identifiés (fig. $17, n^{\circ} 4$ et 9 ), la retouche permet alors la régularisation des bords et/ou une modification de l'angle de tranchant. D'autres pièces (fig. $17, \mathrm{n}^{\circ} 6$ et 8 ) ont été appointées par le biais de la retouche, celle-ci a alors pour rôle de façonner la morphologie générale de la pièce.

La catégorie des outils de type Paléolithique supérieur est peu représentée, les grattoirs comportent des bords retouchés qui forment un front ogival à arrondi (fig. 17, $n^{\circ} 3$ ). 


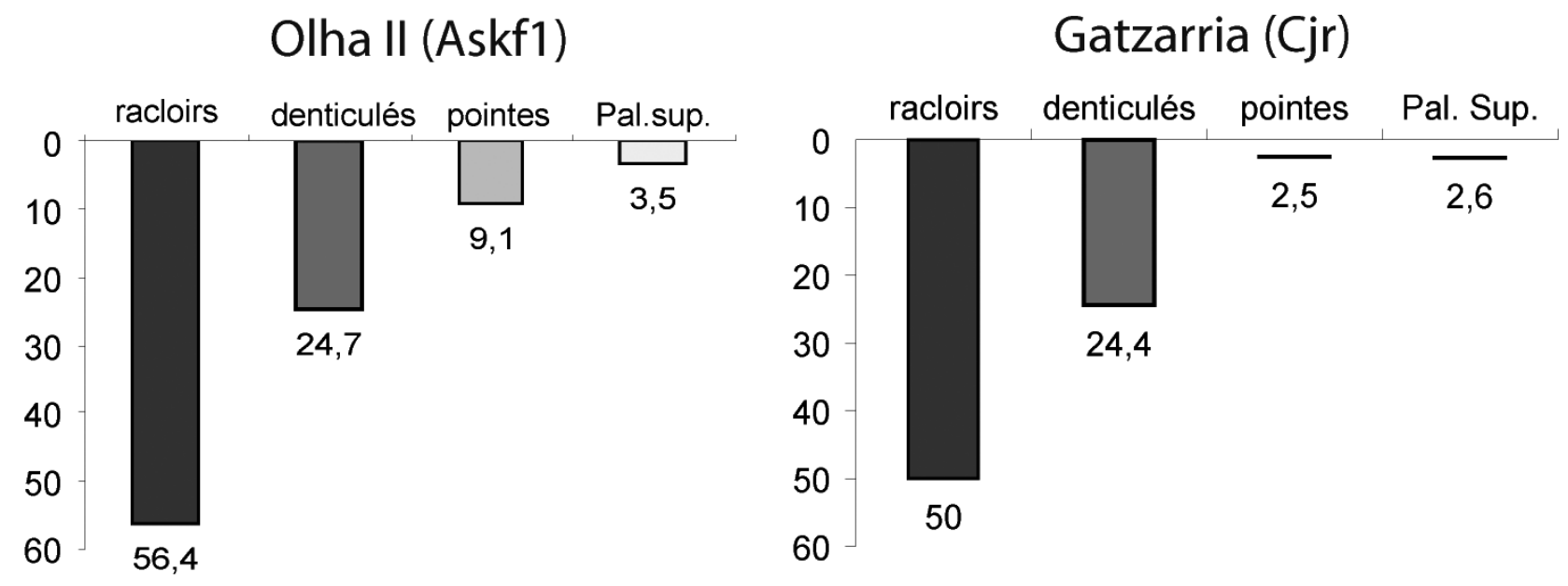

Figure 16 - Proportions des groupes d'outils présents à Olha Il et Gatzarria.

Figure 16 - Tool group's distribution at Olha II and Gatzarria.

\section{Les supports d'outils}

La plupart des outils d'Olha II ont été confectionnés sur éclats. Les éclats à tranchant périphérique sont les supports les plus utilisés dans la confection de l'outillage. Les éclats à dos débordants sont également bien représentés ; les pointes pseudo-levallois (fig. $17, n^{\circ}$ 9) restent par contre assez rares. La sélection des produits débordants semble se distinguer dans la confection de denticulés à Olha II. Des éclats de façonnage de bifaces ont parfois aussi été retouchés à Olha II (fig. 17, $n^{\circ}$ 6). Des éléments appointés ont notamment été confectionnés sur ce type de support mince et rectiligne.

Enfin, les éclats Kombewa (fig. 17, $n^{\circ}$ 8) ont été assez rarement utilisés en tant que support d'outils. Leur présence restreinte aussi au sein des éclats bruts confirme que ces pièces ne constituent pas un des objectifs du débitage en vue de l'obtention d'une pièce à tranchant biconvexe périphérique mais seraient plutôt des produits documentant une phase initiale, au sein du débitage Discoïde, à partir de faces inférieures d'éclats.

\section{Gestion de l'outillage}

Nous avons pu identifier d'assez nombreux éclats de ravivage (fig. 17, $\mathrm{n}^{\circ} 10,11$ ). Au total, 57 de ces pièces ont été identifiées parmi les éclats bruts et les outils. Ces éclats peuvent être divisés en trois catégories :

- la première, et la plus importante numériquement (71,9\%), consiste en des enlèvements profonds et étendus. Ils sont détachés dans le même sens que les éclats de retouche, depuis la face inférieure de l'outil, emportant une partie du bord retouché ;

- la deuxième catégorie (19,3\%) se compose d'éclats de coups de tranchet latéraux sur supports non façonnés (Bourguignon 1992) ;

- enfin, la catégorie la moins représentée (8,8 \%) consiste en des enlèvements longitudinaux dont la face supérieure n'est autre que la face inférieure de l'éclat-support. Nous avons dissocié ces éclats de ceux de la seconde catégorie mais ils peuvent entrer dans la variabilité des coups de tranchets latéraux. Ainsi, la retouche est enlevée et l'angle du tranchant est modifié. On peut en conclure que les tranchants actifs des outils sur éclats sont fréquemment ravivés.

Des chutes de coup de tranchet proviennent aussi de supports façonnés. Plusieurs chutes, provenant de supports façonnés ou non, sont outrepassées, ce type d'accident étant caractéristique de cette action (Bourguignon 1992).

Ce ravivage fréquent peut être lié à des causes fonctionnelles, des causes liées à la durée d'occupation du site ou des facteurs environnementaux. Cependant, ce comportement ajouté au fort degré d'exhaustion des nucléus indique une gestion particulière de la matière première qui pourrait être liée à l'éloignement relatif de la source privilégiée, le silex du Flysch, disponible à une dizaine de kilomètres du site. Cette distance, même si elle est assez limitée (fig. 6) peut entraîner une organisation économique impliquant une durée de vie de l'outillage assez importante et une exploitation exhaustive des nucléus. 

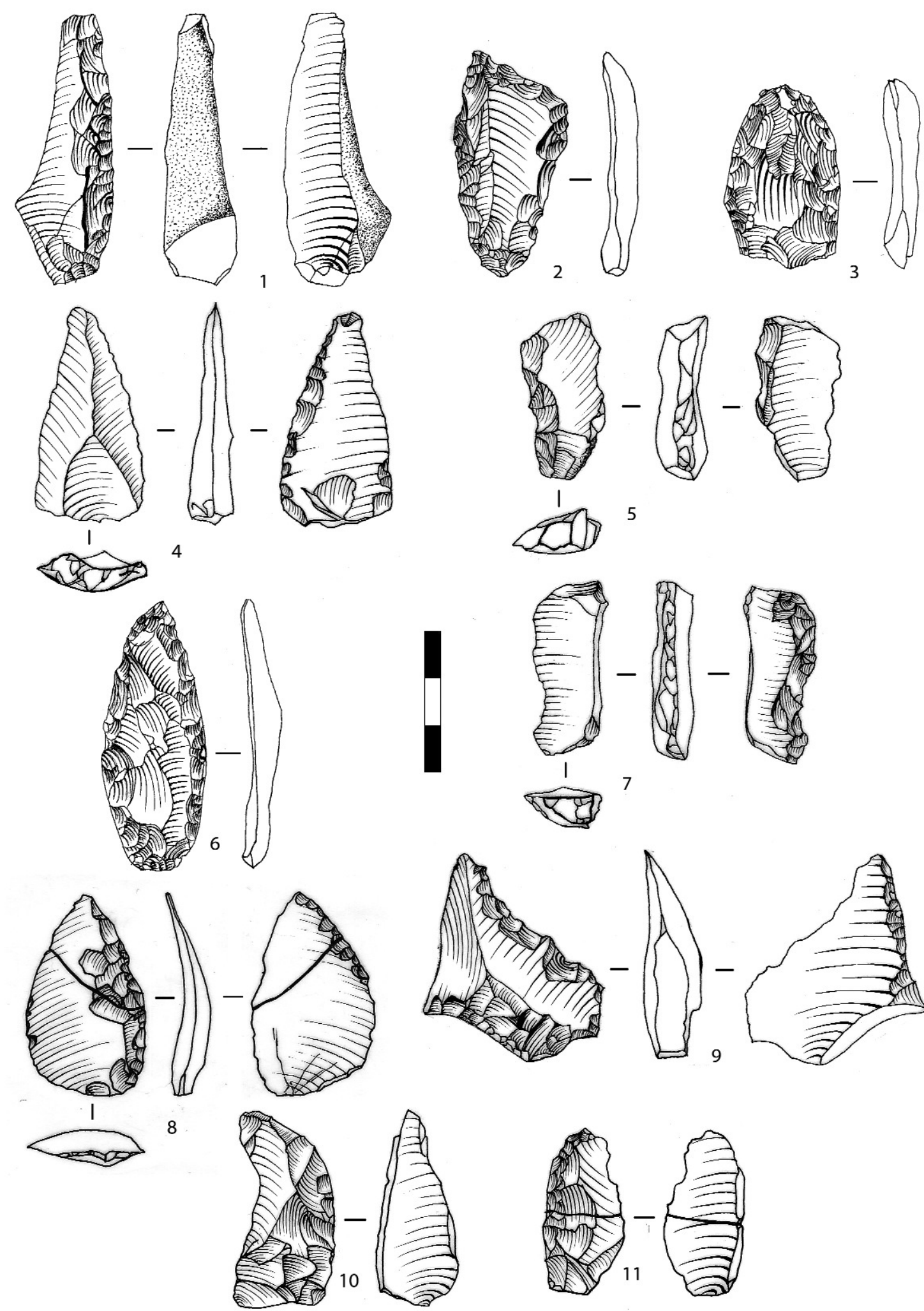

Figure 17 - Outils retouchés. 1 : racloir simple latéral ; 2 : racloir multiple ; 3 : grattoir ; 4, 6 et 9 : pointes ; 5 et 7 : denticulés ; 8 : racloir alterne ; 10 et 11 : coups de tranchet latéraux.

Figure 17 - retouched tools. 1 : Scraper with one retouched edge ;2:Scraper with three retouched edges ; 3 : end-scrapper ; 4, 6 and 9 : points ; 5 and 7 : denticulates ; 8 : alternate sidescrapper ; 10 and 11 : lateral tranchet blow. 


\section{Le macro-outillage}

\section{Les bifaces}

Aucun biface n'a été identifié dans la couche Askf-1 de Olha II, un seul exemplaire provient des premières fouilles de G. Laplace (fig. 18) ; cependant plusieurs éclats de façonnage attestent de la confection de cet outil sur le site. Une première hypothèse est que les bifaces ont été exportés après un façonnage in situ. Cependant, la collection d'Olha I, conservée au Musée de l'Homme, comporte 12 bifaces en silex et 2 en quartzite, de dimensions et de morphologies variables, présentant dans la majorité des cas une base réservée corticale. Ces deux gisements n'étant distants que de quelques mètres, il est possible que les éclats de façonnage d'Olha II et les bifaces d'Olha I soient à mettre en relation. Ainsi, Olha I et || formeraient potentiellement un seul et même site.

Trois groupes morphologiques dominants ont été identifiés : des bifaces amygdaloïdes, des bifaces à tranchant transversaux et des bifaces nommés « bifaces déjetés 》 par E. Passemard (1936). II semble que ce type soit un intermédiaire entre les deux premiers, puisque selon l'orientation de la pièce, elle peut être rapprochée des bifaces à tranchant transversaux ou des bifaces amygdaloïdes (fig. 19). En outre, des mesures réalisées sur les angles d'ouverture des pointes des bifaces déjetés indiquent un angle le plus souvent proche de $90^{\circ}$, ce qui est supérieur à ceux des bifaces amygdaloïdes (fig. 20). Cet angle, observé sur les bifaces déjetés semble correspondre à une rupture morpho-technique entre la partie active et un des bords plutôt qu'à une partie active formée par la rencontre de ces bords. II semble donc plus juste de les rapprocher des bifaces à tranchant transversaux, plutôt que des bifaces amygdaloïdes.

Les différences de morphologie entre ces bifaces pourraient indiquer des différences fonctionnelles. Dans un cas, le façonnage d'un tranchant transversal est recherché, alors que dans l'autre ce sont des bords tranchants en association avec une pointe axiale. Bien que la prise de mesures des angles des tranchants ait été effectuée, celleci n'a pas montré de différences notoires selon les types de pièces. II est donc pour l'instant délicat d'avancer des hypothèses fonctionnelles concernant ces bifaces.

\section{Les hachereaux}

Si la présence de hachereaux est attestée dans chacune des séries étudiées, leur effectif est variable : seulement 4 hachereaux sont présents à Olha II (fig. 21), 7 à Gatzarria et 5 à Isturitz mais nous en avons décompté 83 à Olha $1^{4}$. Cette collection constitue la plus importante série de hachereaux moustériens connue en France. Une étude spécifique a donc été menée à partir de l'ensemble des hachereaux d'Olha I et permet d'amorcer une réflexion sur les raisons de la production de cet pour une période durant laquelle leur utilisation n'est avérée que dans cette région du monde.

Les hachereaux de Olha I (fig. 22) sont majoritairement confectionnés sur ophite $(n=64)$. Cette roche métamorphi- que, proche de la dolérite, est disponible en quantité à proximité immédiate du site, dans le cours de la Nive. Les zones néocorticales étant très fréquentes, c'est probablement dans ce contexte secondaire que le groupe implanté à Olha est venu s'approvisionner. La chaîne opératoire de production des hachereaux n'a pas été entièrement réalisée au sein du gisement, ni sur aucun des sites que nous avons étudiés. Nous supposons donc que les lieux de production se situent sur les lieux d'approvisionnement. Le quartzite est aussi utilisé $(n=13)$; il est ramassé dans les mêmes conditions. Enfin, quelques hachereaux ont été réalisés en calcaire $(n=4)$. Ce calcaire semble être la roche encaissante de l'abri.

Les hachereaux de Olha I présentent un allongement peu important. Ces dimensions peuvent éventuellement avoir été conditionnées par les dimensions des galets présents dans l'environnement (fig. 26).

L'angle des tranchants étudiés se répartit de 26 à $63^{\circ}$, avec une moyenne de $42,2^{\circ}$. La mesure de cet angle est essentielle car il semble qu'il constitue une caractéristique importante dans le mode de fonctionnement de ces pièces (fig. 23).

La grande majorité des hachereaux sont du type 0 selon la typologie de J. Tixier (1956) : le tranchant n'est pas prédéterminé par des éclats antérieurs au détachement de l'éclat-support et la face supérieure est néocorticale (fig. 24). Les faces inférieures présentent un point d'impact majoritairement situé dans l'axe de l'outil. Aucun stigmate de fracturation sur enclume n'est décelable.

Sur les pièces possédant une prédétermination du tranchant, nous avons représenté, sous forme de roses des vents, les directions de débitage des éclats prédéterminant et du détachement de l'éclat-support (Tixier In Balout et al. 1967). Afin de mettre en relation ces deux séries de données, elles ont été regroupées au sein d'un tableau permettant de coupler ces données pièce par pièce, sur le modèle proposé par V. Mourre $(2003,2007)$. Ainsi, l'angle formé par la direction de détachement de l'éclat-support et par la direction des éclats prédéterminants nous permet d'évaluer le degré d'élaboration et de prédétermination de la série. La courbe cumulative obtenue a été superposée à celles de séries de hachereaux acheuléens (fig. 25), seules données disponibles pour le moment (Mourre 2007). Les résultats indiquent que les hachereaux d'Olha I font partie du groupe présentant le degré de prédétermination le plus important : " [...] les hachereaux dont l'éclat préparant le tranchant et l'éclat-support ont été débités dans le même sens représentent un investissement technique et une gestion des volumes minimes, tandis que ceux dont le support et les enlèvements du tranchant sont détachés dans des directions opposées supposent un meilleur contrôle des convexités distales et une gestion plus élaborée des volumes » (Mourre 2007 - p. 69). Ce haut degré de prédétermination se formalise souvent par une standardisation des pièces. Celle-ci est perceptible si l'on compare les dimensions des hachereaux de Olha I à ceux du Castillo et de la grotte Morín (fig. 26) sur la base des travaux de L. Benito del Rey (1972-1973, 1981).

(4) E. Passemard signale l'existence de " plusieurs centaines de ces pièces, dont le poids peut atteindre la livre 》 (1936). II es possible qu'une partie de la collection soit égarée ou qu'elle soit conservée dans un lieu restant à identifier. 

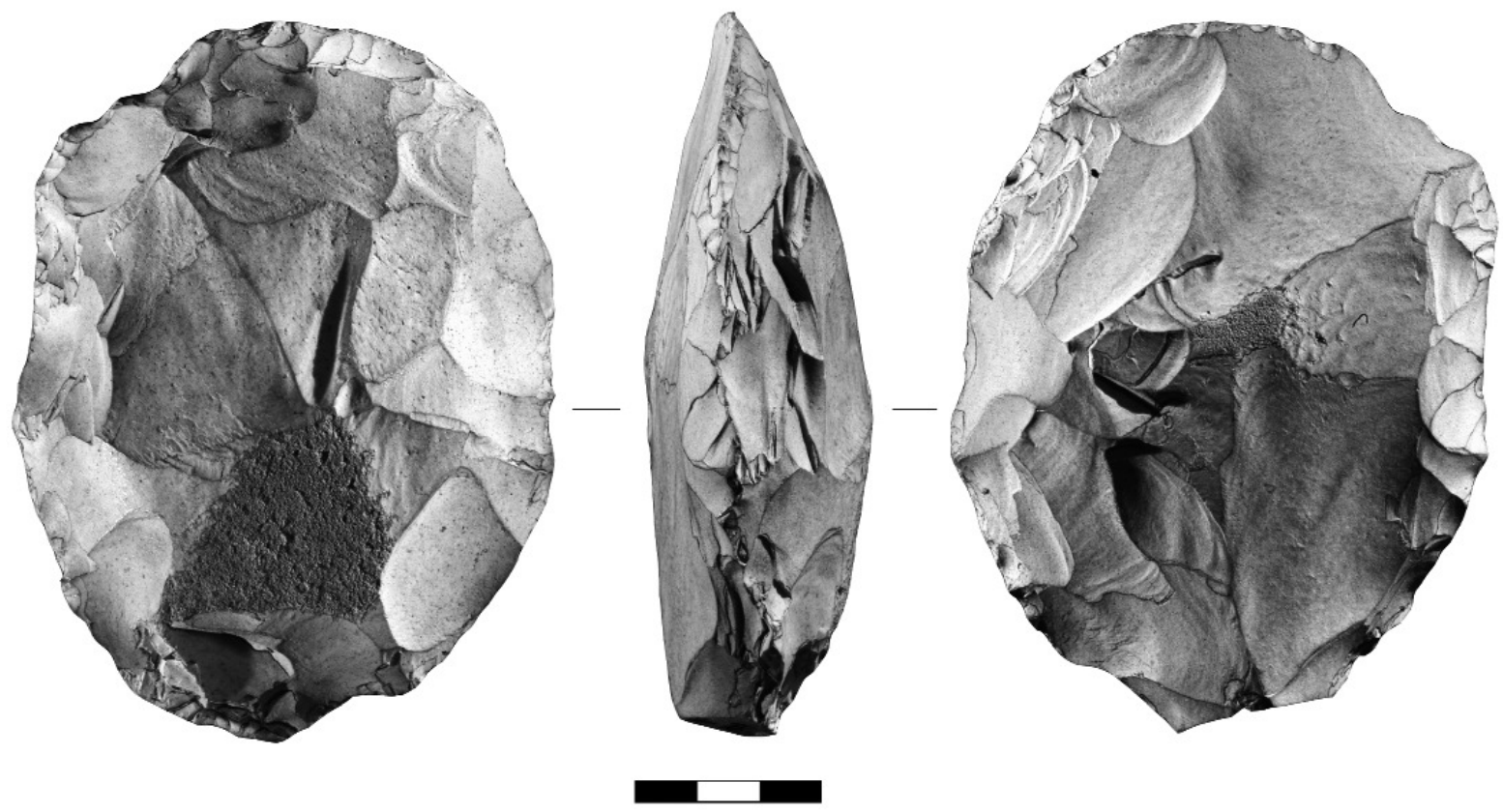

Figure 18 - Biface ovalaire de la couche 2 d'Olha Il, premières fouilles de G. Laplace (1953-1961), photo P. Jugie, MNP. Figure 18 - Oval handaxe from layer 2 at Olha II, first excavations by G. Laplace (1953-1961), photo P. Jugie, MNP.
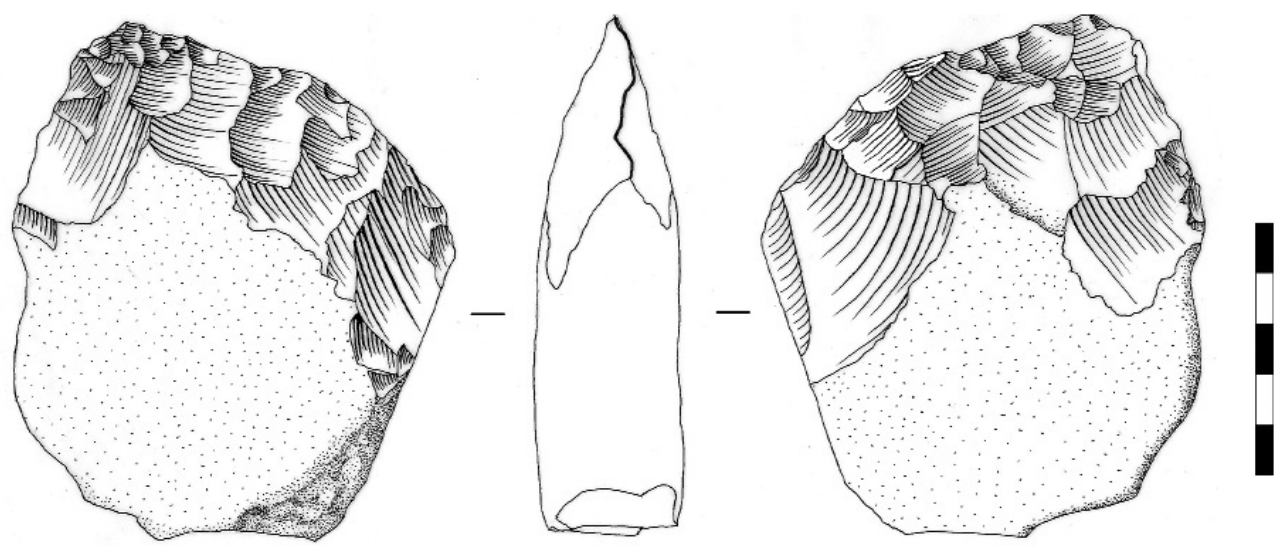

Figure 19 - Biface déjeté provenant de la couche Fi 3 d'Olha I, fouilles E. Passemard (1917-1919).

Figure 19 - Canted handaxe from layer Fi 3 at Olha I, excavation by E. Passemard (1917-1919).

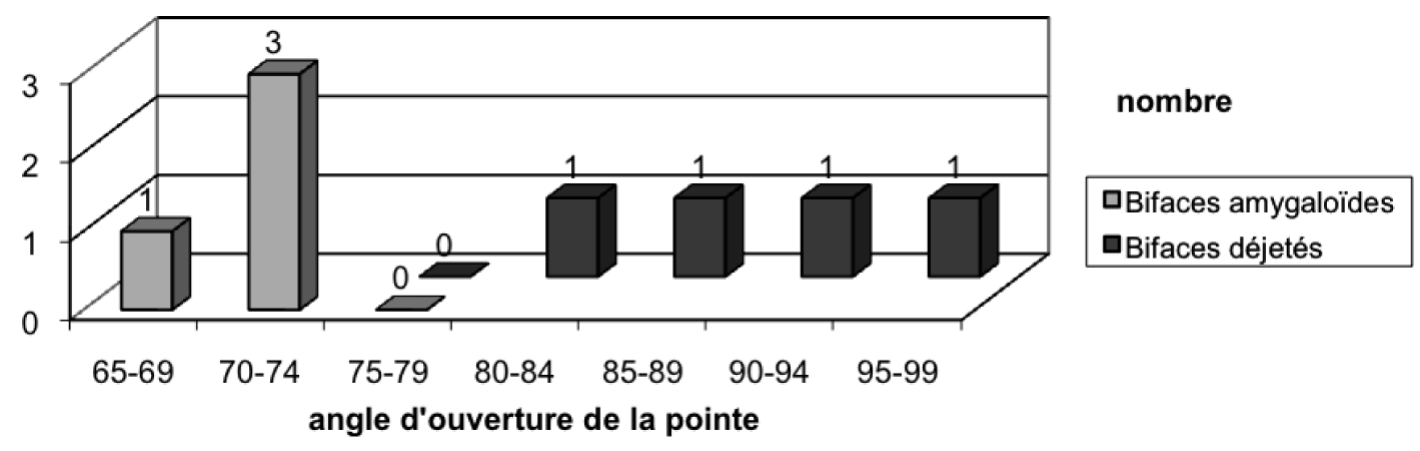

Figure 20 - Comparaison des angles d'ouverture de la pointe des bifaces amygdaloïdes et déjetés.

Figure 20 - Distribution of tips openning angles of almond shaped and déjeté handaxes. 

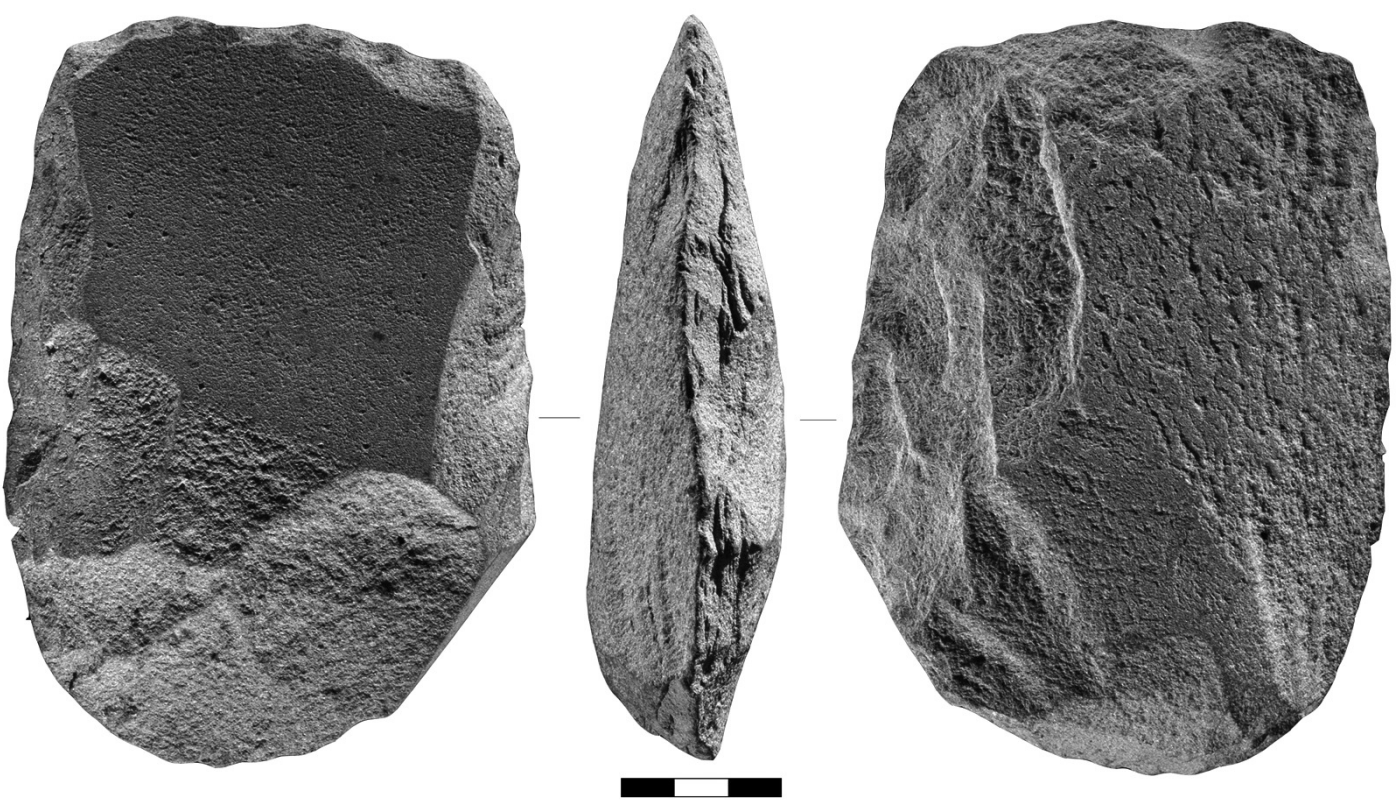

Figure 21 - Hachereau en ophite de type 0 d'Olha II, photo P. Jugie, MNP.

Figure 21 - Ophite cleaver of type 0 at Olha II, photo P. Jugie, MNP.

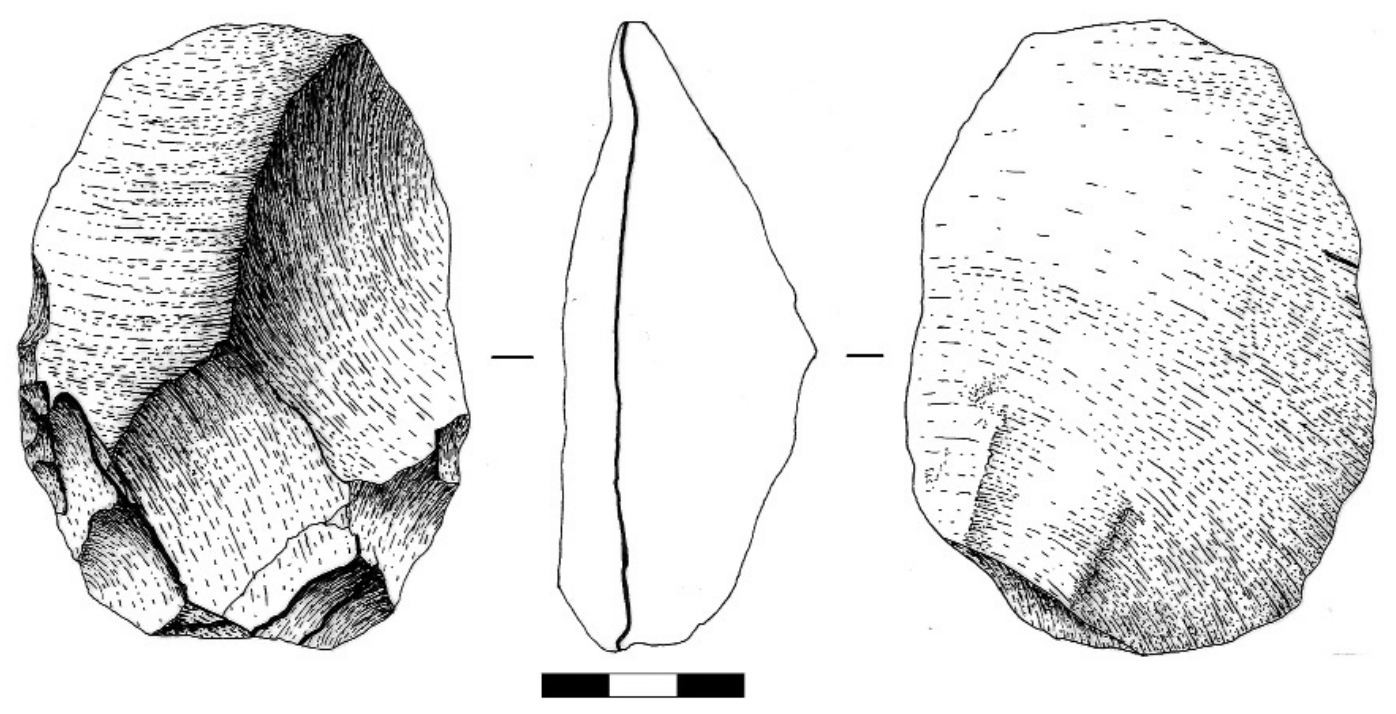

Figure 22 - Hachereau en ophite du type 2 d'Olha I.

Figure 22 - Ophite cleaver of type 2 at Olha I. 


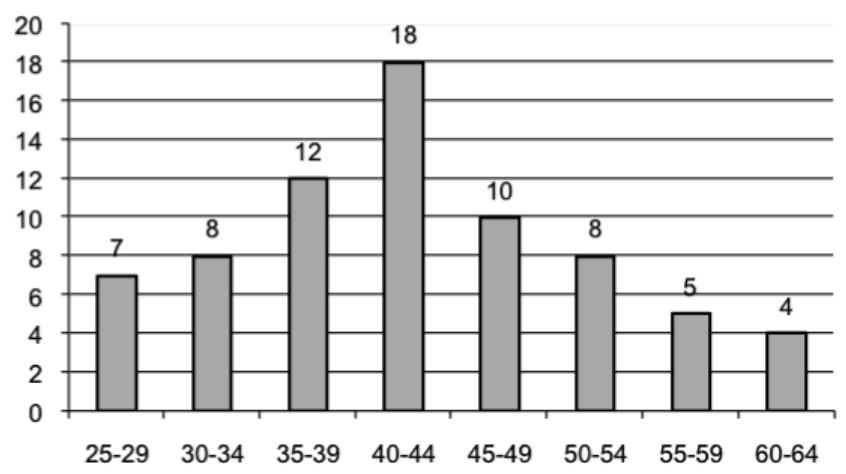

Figure 23 - Répartition des angles de tranchant des hachereaux d'Olha I par classe de 5 degrés.

Figure 23 - Distribution of cleavers cutting edge angles at Olha I by tranches of 5 degrees.

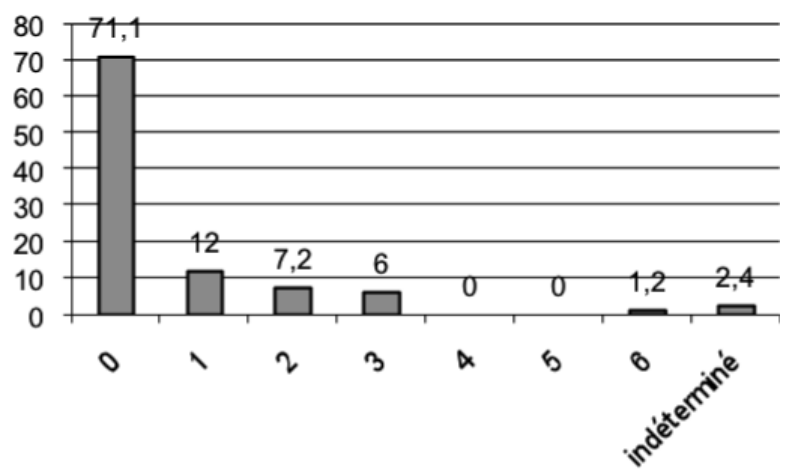

Figure 24 - Répartition des hachereaux d'Olha I par types.

Figure 24 - Distribution of cleaver types at Olha I.

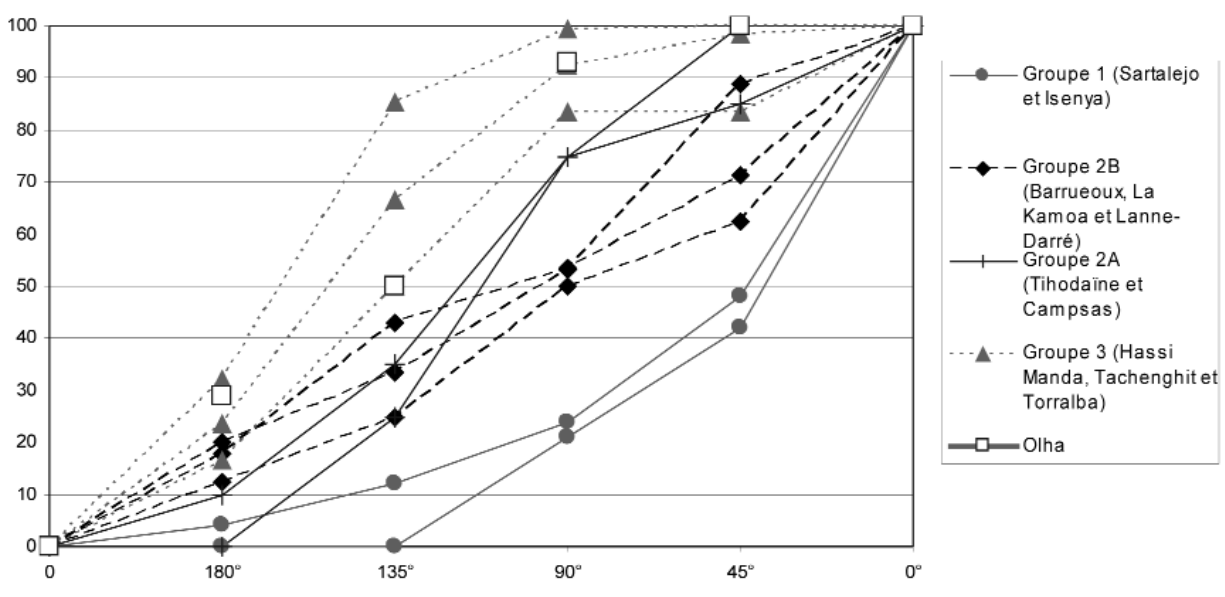

Figure 25 - Comparaison des courbes cumulatives des combinaisons de directions de débitage et des éclats prédéterminants d'Olha l et de séries acheuléennes étudiées par V. Mourre (2003).

Figure 25 - Comparison between cumulative curves combinating flaking direction and predeterminating flakes at Olha I and from acheulean series studied by V. Mourre (2003).

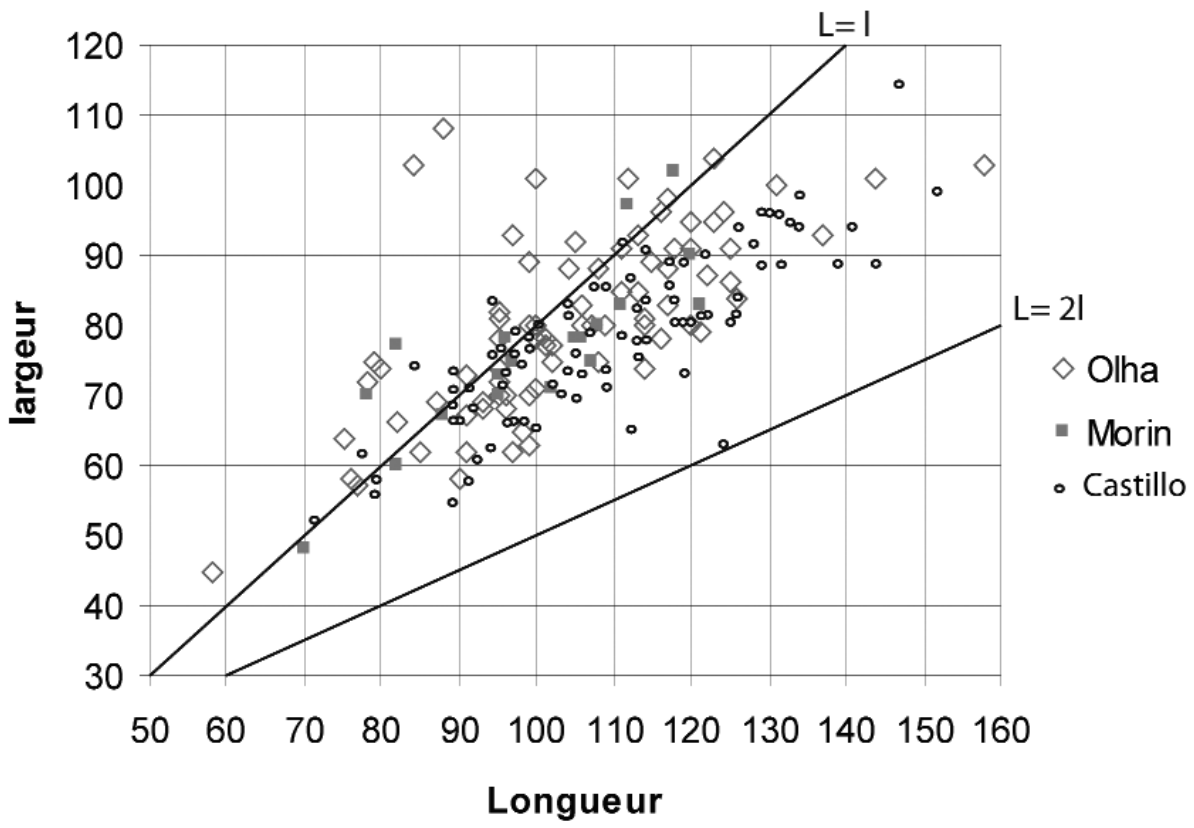

Figure 26 - Comparaison dimensionnelle entre les hachereaux d'Olha, ceux de Morín et du Castillo (D'après Benito del Rey 1972, 1981). Figure 26 - Size distribution of cleavers at Olha I, Morín and Castillo Caves (after Benito del Rey 1972, 1981). 


\section{Bilan et discussion}

\section{Synthèse des éléments caractéristiques}

Les caractéristiques retenues au sein des ensembles analysés sont, en premier lieu, la prépondérance du débitage Discoïde au sein des chaînes opératoires présentes et l'existence d'un débitage prismatique, élément original également attesté dans certains sites de Cantabrie.

L'outillage se caractérise par la prédominance des racloirs, les denticulés constituant le second groupe. La rareté des pointes et des outils de type Paléolithique supérieur est aussi à souligner. Les proportions de l'outillage des séries de Olha Il et Gatzarria présentent des similitudes importantes, que l'on retrouve au sein d'autres séries (cf. infra).

Les hachereaux sont par définition présents dans toutes les séries attribuées au Vasconien. Les bifaces ne sont par contre pas systématiquement présents. L'association des hachereaux et des bifaces est assez variable.

\section{Comparaisons régionales}

Comme cela a été souligné, le concept de débitage Discoïde est dominant sur l'ensemble des séries étudiées. Ce débitage est aussi majoritaire dans l'ensemble des séries de la zone d'étude qui présentent un outillage sur éclat associé à un macro-outillage (hachereaux et bifaces).

Certaines séries présentent aussi une méthode de débitage Levallois comme à la grotte du Noisetier (FréchetAure, Hautes-Pyrénées ; Mourre et Thiébaut 2009) ou à Amalda (Cestona, Guipuzcoa ; Rios 2007). Ce débitage n'est cependant jamais dominant. À Olha II et Gatzarria, le débitage Levallois n'est représenté que par un très faible pourcentage de produits $(1,4 \%)$ sans qu'aucun nucléus ne puisse y être rattaché. La question de la place de ce débitage demeure à préciser dans ces industries.

Le débitage prismatique que nous avons identifié à Olha II et Gatzarria est aussi présent dans plusieurs séries des Monts cantabriques puisque J.-M. Maillo Fernandez l'a signalé à la grotte Morín et au Castillo (Maillo Fernandez et al. 2004).

En ce qui concerne le petit outillage retouché sur éclat, les différentes études typologiques anciennes rendent parfois délicates les comparaisons. L'étude de V. Cabrera Valdès sur l'industrie du Castillo (1984) mentionne des proportions de l'outillage très proches de celles d'Olha II et de Gatzarria (50,5\% pour les racloirs ; 21,5\% pour les denticulés ; $6 \%$ pour les pointes; 4,8 \% pour les outils de type Paléolithique supérieur). L'étude récente de l'industrie de la Grotte du Noisetier (racloirs : 46,4 \% ; denticulés : $32,1 \%$; pointes : 10,7\% ; autres : 10,7\% ; Mourre et al. en cours) indique aussi des pourcentages similaires. À Amalda (Rios 2007), l'outillage est dominé par les racloirs, suivis par les denticulés. Nous ne connaissons pas les proportions exactes des outils pour l'ensemble des matières premières. Cependant, pour le silex (qui constitue $85,4 \%$ de la matière première), les racloirs représentent $59 \%$ de l'outillage ; les denticulés $23,8 \%$; les pointes : 7,6\% ; autres : $3,8 \%$ ).
L'association des bifaces et des hachereaux dans le Moustérien vasco-cantabrique a été identifiée au sein de plusieurs sites. Pour l'OIS 3, on connaît le site du Noisetier (Mourre 2006), Harregi (Saenz de Buruaga et Ebrard 2004), Amalda (Rios 2007), Abauntz (Mazo et Utrilla 1996 ; Arrizabalaga 2005) ou encore le Castillo (Cabrera Valdès 1984). La station de surface de Calavanté (HautesPyrénées ; Sacco-Sonador 2008) présente aussi cette association, mais il est impossible à l'heure actuelle de caler cette série chronologiquement. De rares séries rapportées à la fin de l'OIS 5 livrent aussi le couple hachereau/biface. C'est le cas de la Verde III et l'Ermita del Abra, rapportés relativement au stade 5.5 sur la base de travaux géo-archéologiques (Montes Barquin 1994 ; Guttierrez Morillo et Serrano Canadas 2004). Comme le note H. Djema, " La similitude observée entre ces sites appartenant à deux stades isotopiques différents (M.I.S. 5.5. pour la Verde ; M.I.S. 3. pour Esquilleu et El Habario), nous interroge sur la présence de traditions régionales fortes, sur la validité des datations relatives, sur la recherche de distinction entre ces périodes au sein d'autres domaines (gestion du territoire, mobilité, économie, etc.), sur le rôle que joue le contexte environnemental et pétrographique au sein de ces séries ! ”(Djema 2008).

La proportion des hachereaux et des bifaces varie de façon importante selon les séries. Les bifaces sont même absents de certaines séries où les hachereaux sont attestés, comme à la grotte Morín (Gonzalez Echegaray et Freeman 1973) ou à El Pendo (Gonzalez Echegaray 1980). À l'inverse, quelques rares séries possèdent uniquement des bifaces et ont été attribuées au Moustérien de Tradition Acheuléenne, comme le site de Murba (Baldeon 1990) ou le Basté (Chauchat et Thibault 1968). Quelle peut être la signification de cette importante variation numérique selon les sites ? Pour répondre à cette question quantitative, il est nécessaire de réfléchir, en premier lieu, sur la signification de leur présence d'un point de vue qualitatif.

\section{Signification de la production de hachereaux}

Les hachereaux produits dans le Moustérien de la région franco-cantabrique soulèvent de nombreuses questions, notamment parce que leur production récurrente n'est documentée que dans cette aire géographique et à cette période. Diverses hypothèses peuvent être émises concernant les raisons de cette production particulière.

Les premiers fouilleurs à s'être interrogés sur ces raisons sont H. Obermaier (1924) et R. de la Vega del Sella (1921). Leurs travaux concernent respectivement les gisements du Castillo et de la grotte Morín. Ils défendent l'hypothèse selon laquelle les hachereaux moustériens se seraient perpétués depuis l'Acheuléen en Espagne. II semble cependant difficile de parler de "survivance " (Vega del Sella 1921) puisque la présence de hachereaux dans les niveaux moustériens cantabriques reste ponctuelle. Ils ne sont présents, le plus souvent, que dans un seul niveau au sein des différents gisements (sauf à la grotte Morín et l'abri Olha I), toujours précédé en stratigraphie par d'autres niveaux moustériens n'en possédant pas, ce qui montre que cet outil a été abandonné à certains moments, puis reproduit dans un contexte entièrement différent. Par exemple, à la 
grotte du Castillo, les hachereaux sont absents des niveaux acheuléens ainsi que des niveaux moustériens sous-jacents à la couche 20. Ainsi, la production des hachereaux ne peut être considérée comme une « survivance » depuis l'Acheuléen dans la région.

Une autre hypothèse consiste à mettre en avant le poids du déterminisme environnemental dans cette production. Les hachereaux seraient préférentiellement produits et utilisés dans des environnements pauvres en silex de bonne qualité. Pourtant, à l'abri Olha, la majorité de l'industrie est réalisée en silex du Flysch et des bifaces de dimensions variables sont réalisés dans cette matière. Les hachereaux sont réalisés sur ophite ou quartzite ; un approvisionnement spécifique existe donc en vue de leur production même dans un contexte où le silex de bonne qualité permet la fabrication d'autres outils massifs tels que les bifaces.

L'explication fonctionnelle peut également être envisagée. La morphologie générale des hachereaux indique que ces outils ont pu intervenir dans une gamme d'utilisation nécessitant une forte résistance du tranchant. Cependant, peu d'études tracéologiques ont été menées sur des hachereaux. Une analyse a concerné des hachereaux acheuléens de la vallée de la rivière Najerilla (Utrilla \& Mazo 1996), mais les pièces analysées proviennent d'un contexte incertain (ramassage de surface) et leur nombre est trop restreint pour en tirer des interprétations (3 hachereaux en quartzite possèdent des micro-polis de bois ; 1 autre présente un poli très peu développé de bois ou d'os ; 1 seul hachereau en silex présentait des traces d'usure, liées à la découpe de la viande). Plus récemment, les hachereaux moustériens du niveau VII du site d'Amalda (Cestona, Guipuzcoa, Espagne) ont été étudiés par J. Rios qui propose leur utilisation en coupe ou en percussion dans des activités de gros-œuvre sur la base de macro-traces (Rios 2007). Si des approches tracéologiques ont été réalisées sur le quartzite (Plisson 1986), aucune expérimentation spécifique à ce type d'outil n'a été effectuée afin d'en évaluer les fonctions potentielles. Le peu d'études portant sur la fonction des hachereaux restreint pour le moment les possibilités d'interprétations. Pourtant, si cet outil n'est plus confectionné depuis l'Acheuléen, d'autres types d'outils ont servi pour accomplir les différentes activités menées par les groupes humains. Ainsi, le déterminisme fonctionnel ne peut être une hypothèse satisfaisante pour expliquer les raisons de production de cet outil dans cette zone.

En procédant par élimination, on peut finalement conclure que cette production est un choix délibéré de la part d'un groupe et que leur présence ne peut donc pas être liée à un déterminisme environnemental ou fonctionnel. Ainsi, on peut considérer que ces outils constituent un marqueur culturel d'un groupe au sein du Moustérien vasco-cantabrique (fig. 27). Cette entité est restreinte géographiquement ; c'est précisément ce qui en fait un ensemble important au sein de la problématique concernant les raisons et les implications de la diversification des industries au Paléolithique moyen.
Afin d'évaluer la part des motivations fonctionnelles dans la production des hachereaux, des expérimentations visant à cerner leur(s) fonction(s) sont en cours avec la collaboration d'É. Claud, D. Colonge, V. Mourre et C. Servelle dans le cadre du PCR intitulé "Des Traces et des Hommes: Projet de recherche interdisciplinaire sur l'identification des modalités d'acquisition et de traitement des matières animales et végétales au Paléolithique moyen en Europe occidentale », coordonné par $\mathrm{C}$. Thiébaut.

\section{Réflexions sur les matières premières}

L'ensemble des analyses effectuées sur les industries de Gatzarria, d'Isturitz et d'Olha I et II met en exergue un aspect économique particulier au sein de ces industries. Deux types de comportement sont visibles :

- d'une part, on a une chaîne opératoire entièrement effectuée sur le site. Le débitage Discoïde a été mené sur plusieurs matières premières (silex à Olha, quartzites à Gatzarria et secondairement la lydienne et le quartz à Gatzarria) qui possèdent différentes aptitudes à la taille. C'est donc une méthode de débitage qui présente une grande adaptabilité à son environnement minéral.

- d'autre part, un débitage lié à la production de hachereaux est effectué hors des sites (sur les aires d'approvisionnement ?) et seuls les outils finis sont importés. Ce débitage présente une spécialisation importante, à travers une sélection rigoureuse des blocs liée à un projet technique spécifique et à la recherche de matériaux aux propriétés mécaniques particulières : l'ophite est exclusivement dévolue à cette production.

II existe donc une dialectique économique entre une chaîne opératoire adaptée à son environnement minéral et une autre qui possède un haut degré de spécialisation dans la sélection de la matière et la technique employée.

Si le débitage Discoïde peut être considéré comme une forme hautement adaptative à son environnement, il n'est cependant pas spécifiquement appliqué à certaines matières ayant des propriétés mécaniques réputées médiocres. Effectivement, il a parfois été envisagé que celui-ci était préférentiellement employé comme alternative au débitage Levallois dans des zones où la qualité de la matière première pourrait nécessiter l'utilisation d'un schéma conceptuel plus souple en terme de prédétermination. Cette hypothèse ne peut être validée si on examine le Moustérien des Pyrénées puisqu'à Olha, le débitage Discoïde est mené sur silex du Flysch, matière qui présente une bonne aptitude à la taille. Parallèlement, le débitage Levallois est employé dans des environnements où le silex fait défaut comme à la grotte du Noisetier, où il est mené sur quartzite. L'environnement minéral ne détermine donc pas l'utilisation préférentielle de l'un ou l'autre concept.

\section{Chronologie et environnement}

Le champ d'investigation concernant la chronologie de ce faciès reste à préciser. En effet, plusieurs types de données sont en contradiction concernant la datation de ce faciès : 


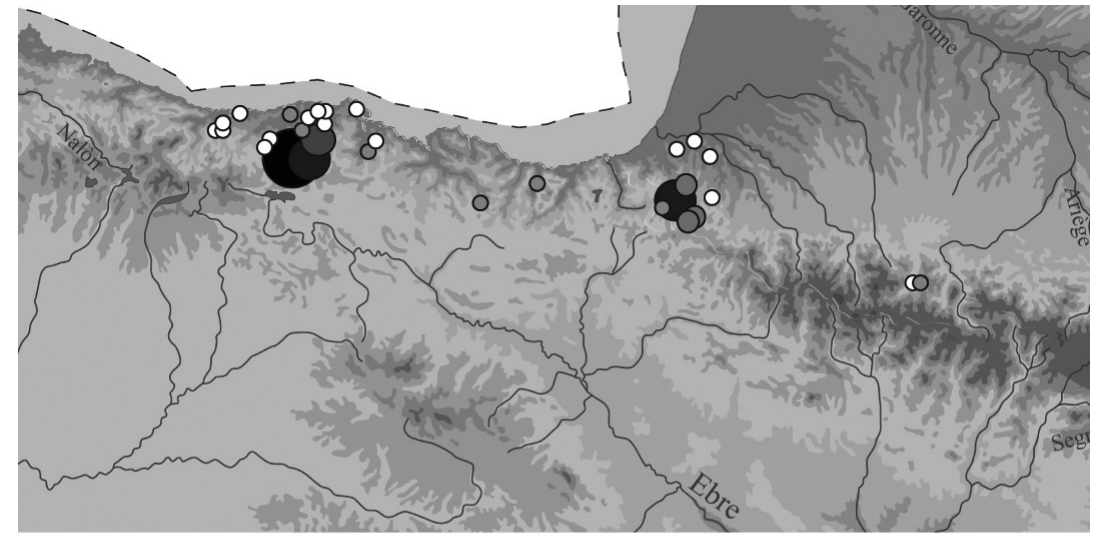

○ Hachereaux trouvés en surface associés à une industrie Moustérienne

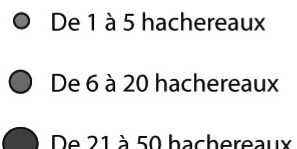

De 21 à 50 hachereaux

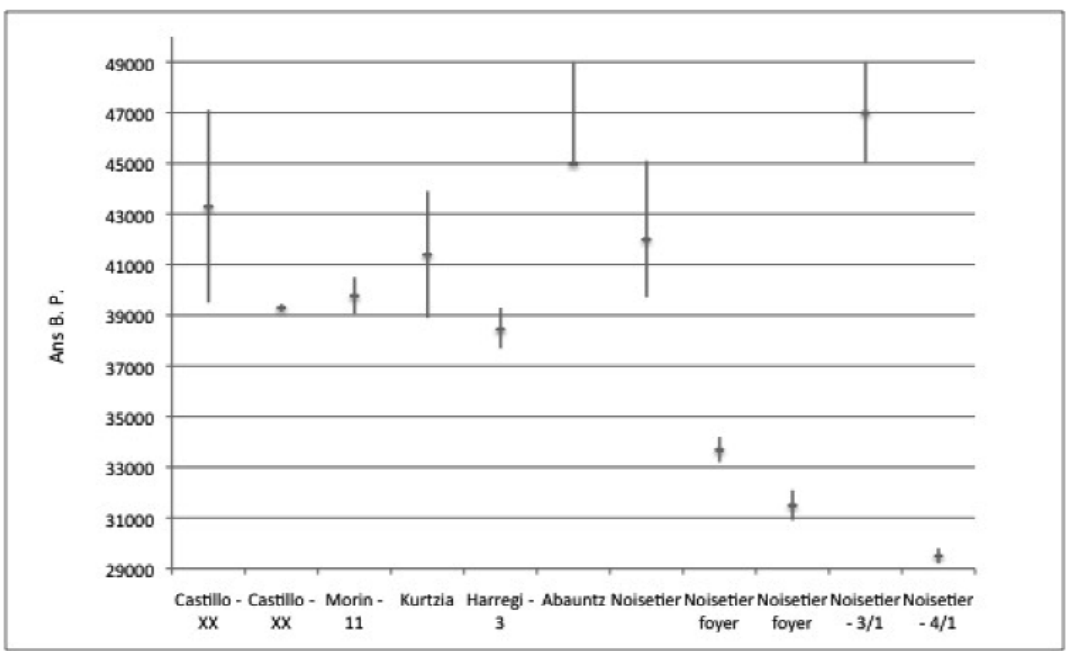

Figure 27 - Répartition des hachereaux en contexte moustérien dans la zone Vascocantabrique.

Figure 27 - Mousterian cleavers distribution in Vasco-cantabric area.
Figure 28 - Datations absolues (C14) de plusieurs couches moustériennes contenant des hachereaux d'après Allard 1993 ; Cabrera et al 1996 ; Maillo Fernandez 2004 ; Mourre 2005, 2006 ; Muñoz et al .1990; Saenz de Buruaga 2004 ; Utrilla et al. 2000.

Figure 28 - Radiocarbon dates from several mousterian layers containing cleavers. In : Allard 1993 ; Cabrera et al 1996 ; Maillo Fernandez 2004 ; Mourre 2005 et 2006 ; Muñoz et. al. 1990 ; Saenz de Buruaga 2004 ; Utrilla et al. 2000.
- les quelques datations par la méthode du carbone 14 qui ont été obtenues pour des niveaux moustériens contenant des hachereaux donnent des âges proches de 40000 ans BP (fig. 28). II convient toutefois de souligner que la méthode est ici proche de la limite de son champ d'application même si en Espagne les datations récentes pour les occupations moustériennes sont fréquentes ;

- le spectre faunique des niveaux vasconiens d'Olha II et Gatzarria indique la présence d'une faune tempérée dominée par le Cerf (Cervus elaphus), accompagné fréquemment par les bovidés (Bos primigenius) et le Cheval (Equus) (Lavaud 1980). Cette association faunique est aussi présente à la grotte du Noisetier (Mourre 2006), à Amalda (Rios 2007) et au Castillo (Cabrera Valdès et al. 2005). La présence de dents de Rhinoceros merckii à l'abri Olha ainsi qu'à la grotte Morín (Passemard 1936) et dans la couche XX du Castillo (Cabrera et al. 2005) semble confirmer cette attribution à un climat tempéré. Cette association faunique indique, d'après certains auteurs, que ces niveaux seraient plutôt contemporains de la fin du stade isotopique 5 (Jaubert 2007).

De nouveaux travaux concernant les associations fauniques et les modes de subsistance au sein des sites d'Olha I et II vont être entrepris par M.-C. Soulier (Doctorante, Université Toulouse-le Mirail). Cette étude archéozoologique pourra apporter des précisions quant à la position chronologique du Vasconien et de son environnement.

\section{Conclusion}

L'analyse technologique menée à Olha II et Gatzarria montre l'unité des choix techniques effectués sur ces sites. Si des relations peuvent déjà être établies sur des bases 
bibliographiques, l'étude de plusieurs séries provenant des Monts cantabriques est en cours afin de tester l'hypothèse d'un ensemble de traditions techniques et culturelles allant du sud de l'Aquitaine jusqu'aux Monts cantabriques. II est prévu d'effectuer des comparaisons directes avec la couche XX de la grotte du Castillo, la couche XIII de EI Pendo, ainsi que les couches 17 sup. à 13 de la grotte Morín. L'interprétation culturelle du Vasconien s'appuie sur une cohérence certaine, perceptible à travers les résultats obtenus par le biais de l'analyse technologique des ensembles lithiques. En ce qui concerne la production de hachereaux, leur statut de marqueur culturel devra être étayé, mais cette interprétation reste pour l'instant la plus plausible. Nous espérons que les expérimentations à venir nous permettront aussi d'évaluer la part du facteur fonctionnel dans la production de ces outils.

Au-delà de ce travail sur les expressions techniques de ces populations néandertaliennes, c'est une dynamique de recherche collective qui se met en place notamment par le biais d'une approche expérimentale, une étude tracéologique et une étude archéozoologique. La mise en commun des résultats de ces différents travaux devrait permettre d'aboutir à une vision plus fine des modes de vie de ces groupes. L'objectif-clé de cette démarche sera d'argumenter la diversification culturelle perceptible au Paléolithique moyen récent en Europe de l'Ouest et ses implications.

\section{Remerciements}

Cet article est l'aboutissement d'un mémoire de Master II pour lequel je remercie mes tuteurs, F. Bon et V. Mourre. Mes remerciements s'adressent à J-J. Cleyet-Merle, conservateur au Musée national de Préhistoire, ainsi qu'à toute l'équipe du Musée National de Préhistoire pour m'avoir facilité l'étude des collections d'Olha II et de Gatzarria. Je tiens aussi à remercier F. Semah pour m'avoir autorisé à étudier la collection d'Olha I ainsi que l'équipe du Musée de l'Homme. J'exprime aussi ma reconnaissance à Catherine Schwab qui m'a permis d'étudier une partie d'Olha I et Isturitz au Musée d'Archéologie Nationale. La poursuite de ces travaux va être réalisée avec le soutien d'une allocation de formation et de recherche attribuée par le ministère de la Culture et de la Communication dans la région Aquitaine pour l'année 2009. Merci à K. Douze pour ses traductions.

\section{Références bibliographiques}

ALLARD M. 1993 - Fréchet-Aure, Grotte du Noisetier. Bilan scientifique 1992 de la Direction Régionale des Affaires culturelles de Midi-Pyrénées, Ministère de la Culture.

ARRIZABALAGA A. 2005 - Las primeras ocupaciones humanas en el Pirineo Occidental y Montes Vascos. un estdo de la cuestion en 2005, Munibe, Homenaje al Prof. Jesus Altuna, $\mathrm{n}^{\circ}$ 57, p. 5-22.

BALDEON A. 1990 - El Paleolitico Inferior y Medio en el Pais Vasco. Una aproximacion en 1990, Munibe, 42, p. 11-22.
BALOUT L., BIBERSON P. et TIXIER J. 1967 - L'Acheuléen de Ternifine (Algérie), gisement de l'Atlanthrope, L'Anthropologie t. 71, n 3-4, p. 217-237.

BENITO DEL REY L. 1972-1973 - Los hendidores de la capa musteriense "Alfa" de la Cueva del Castillo (Santander). Estudio tipológico, Zephyrus, XXIII-XXIV, p. 269-290.

BENITO DEL REY L. 1981 - Aspectos técnicos y typológicos que relacionan estrechamente el Musteriense con hendidores de las cuevas del Castillo y Morín (Santander), Munibe, 33, n 3-4, p. 157-170.

BINFORD L. R. et BINFORD S. 1966 - A preliminary analysis of functional variability in the Mousterian of Levallois facies, American Anthropologist, vol. 68, 2, p. 238-295.

BOËDA E. 1993 - Le débitage Discoïde et le débitage Levallois récurrent centripète, Bulletin de la Société Préhistorique Française., t. 90, n 6, p. 392-404.

BORDES F. 1953 - Essai de classification des industries " moustériennes ", Bulletin de la Société Préhistorique Française., t. L, $\mathrm{n}^{\circ}$ 7-8, p. 457-466.

BORDES F. et BOURGON M. 1951 - Le complexe moustérien : Moustériens, Levalloisien et Tayacien. L'Anthropologie t. 55, p. 1-23.

BOURGUIGNON L. 1992 - Analyse du processus opératoire des coups de tranchet latéraux dans l'industrie moustérienne de l'abri du musée (les Eyzies-de-Tayac, Dordogne). Paléo n 4, p. 69-89.

BOURGUIGNON L. 1997 - Le Moustérien de type Quina : nouvelle définition d'une entité technique, Université de Paris X - Nanterre, $672 \mathrm{p}$.

CABRERA VALDES V. 1983 - Notas sobre el Musteriense cantábrico : el « Vasconiense », Homenaje al Prof. Martín Almagro Basch, vol. 1, Madrid, Ministerio de Cultura, p. 131-141.

CABRERA VALDES V. 1984 - El yacimiento de la cueva del Castillo (Puente Viesgo, Santander), Bibliotheca Praehistorica Hispana, XXII.

CABRERA VALDES V., BERNALDO DE QUIROS F., MAILLO J-M., PIKE-TAY A., GARRALDA D. 2005 Excavaciones en El Castillo : Veinte años de reflexiones. Neandertales cantabricos, estado de la cuestion, Museo nacional y centro de investigacion de Altamira, p. 505-526.

CABRERA VALDES V., VALLADAS H., BERNALDO DE QUIROS, F., HOYOS GOMEZ M. 1996 - La transition Paléolithique moyen - Paléolithique supérieur à El Castillo, Cantabrie : nouvelles datations par le carbone-14, Comptes rendus de l'Académie des sciences de Paris, 322, p.1093-1098.

CHAUCHAT C. 1985 - L'abri Olha (Pyr. Atl.) - la nouvelle étude de la collection Passemard, Bulletin de la Société Préhistorique Française. t. 82, p. 237-238. 
CHAUCHAT C. et THIBAULT C. 1968 - La station de plein air du Basté, à Saint-Pierre d'Irube (Basses - Pyrénées). Géologie. Etude archéologique préliminaire, Bulletin de la Société Préhistorique Française., vol. 75, n 10, p. 314-326.

DELPORTE H. 1974 - Le Moustérien d'Isturitz d'après la collection Passemard (Musée des Antiquités Nationales), Zephyrus, XXV, p. 17-43.

DESCHAMPS M. 2008 - Le Vasconien et sa signification au sein des faciès moustériens, Université de Toulouse II - le Mirail, Mémoire de Master II, $62 \mathrm{p}$.

DESCHAMPS et MOURRE sous presse - Le Vasconien, un demi-siècle après sa définition par François Bordes. $134^{\text {ème }}$ Congrès national des sociétés historiques et scientifiques, colloque international François Bordes. Editions CTHS.

DJEMA H. 2008 - Le Paléolithique moyen ancien de la corniche cantabrique et du bassin Aquitain ou le phénomène culturel des premiers néandertaliens, Thèse de doctorat, Université Panthéon-Sorbonne (Paris), p. 424.

FREEMAN L. G. 1966 - The nature of Mousterian facies in Cantabrian Spain, American Anthropologist.

GONZALEZ ECHEGARAY J. 1971 - Cueva Morín, Excavaciones 1966 - 1968, Publicaciones del Patronato de las cuevas prehistoricas de la provincia de Santander, p. 445.

GONZÁLEZ ECHEGARAY J. 1980 - El yacimiento de la cueva de "El Pendo" (Excavaciones 1953-57), Madrid, Consejo Superior de Investigaciones Científicas, Instituto Español de Preistoria, p. 270.

GONZÁLEZ ECHEGARAY J. et FREEMAN L. G. 1973 Cueva Morín, excavaciones 1969, Santander, Publicaciones del patronato de las cuevas préhistoricas de la provincia de Santander, $304 \mathrm{p}$

GUTTIERREZ MORILLO A. et SERRANO CANADAS E. 2004 - El Yacimiento del Paléolitico medio de la "Ermita des Abra (Campoo de suso, Cantabria). Aproximacion cultural, chronologica y geomorphologica, Cuaternario y Geomorphologia, Vol. 12 (3-4), p. 27-39.

JAUBERT J. 2007 - Quels peuplements avant l'Aurignacien sur le versant nord des Pyrénées ? In : Qui est I'Aurignacien ? Colloque d'Aurignac, 20-21 septembre 2003, Éditions Musée-forum Aurignac, Cahier 3, pp. 9-25.

LAPLACE G. 1957 - Typologie analytique. Application d'une nouvelle méthode d'étude des formes et des structures aux industries à lames et lamelles, Quaternaria, p. $133-164$.

LAPLACE G. 1986 - À propos des gisements du Pays Basque, Bulletin de la Société Préhistorique Française. t. $83, n^{\circ} 4$, p. $106-112$.
LAPLACE G. 2000 - Application de la typologie analytique et structurale à l'étude de l'outillage moustéroïde de l'abri Olha 2 à Cambo (Kanbo) en Pays Basque. Paleo n 12.

LAPLACE G., MEROC L. 1954 - Application des coordonnées cartésiennes à la fouille d'un gisement, Bulletin de la Société Préhistorique Française., T.LI, p. 58-66.

LAPLACE G. et SAENZ DE BURUAGA A. 2000 Application de la typologie analytique et structurale à l'étude de l'outillage moustéroïde de l'abri Olha II à Cambo (Kanbo) en Pays Basque, Paléo, n 12, p. 261-324.

LAPLACE G., SAENZ DE BURUAGA A. 2002 - Typologie analytique et structurale des complexes du Moustérien de la grotte de Gatzarria(Ossas-Suhare, Pays Basque) et de leurs relations avec ceux de l'abri Olha II (Cambo, Pays Basque), Pyrenae, 33-34, p. 81-163.

LAVAUD F. 1980 - Les faunes paléolithiques du Würm II et III dans le sud-ouest et le centre-ouest de la France, Université de Poitiers.

MAÍLLO FERNÁNDEZ J. M. 2007 - Aproximacion tecnologica del final del Mustériense de Cueva Morin (Villanueva de Villaescusa, Cantabria, España), Munibe, 58, p. 13-42.

MAÍLLO FERNÁNDEZ J. M., CABRERA-VALDÈS, V., BERNALDO DE QUIROS F. 2004 - Le débitage lamellaire dans le Moustérien final de Cantabrie (Espagne) : le cas de El Castillo et de Cueva Morin, L'Anthropologie, 108, p. 367393.

MAZO C. et UTRILLA P. 1996 - Excavaciones en la cueva de Abauntz (Arraiz). Campanas de 1994 y 1995, Trabajos de Arqueologia Navarra, vol. 12, p. 270-279.

MONTES BARQUIN R., MUNOZ FERNANDEZ E., SANGUINO J. 1994 - El yacimiento paleolitico de la Verde (Herrera de Camargo, Cantabria) : problematica de la excavacion arqueologica en un suelo edafologico, in Actas de la 2a Reunion de Geoarqueologia, I.T.G.E. (éd.), Madrid.

MOURRE V. 2003 - Implications culturelles de la technologie des hachereaux, Université de Paris X - Nanterre, Thèse de Doctorat, 3 vol., 880 p.

MOURRE V. 2007 - Émergence et évolution de la prédétermination au Paléolithique. Normes techniques et pratiques sociales : " de la simplicité des outillages pré- et protohistoriques », XXVle Rencontres Internationales d'Archéologie et d'Histoire d'Antibes, 20 - 22 octobre 2005, AntibesJuan-les-Pins.

MOURRE V. (dir) 2005 - Le site moustérien de la Grotte du Noisetier à Fréchet-Aure (Hautes-Pyrénées), premier rapport intermédiaire, Fouille programmée pluriannuelle 20052007.

MOURRE V. (dir) 2006 - Le site moustérien de la Grotte du Noisetier à Fréchet-Aure (Hautes-Pyrénées), Deuxième rapport intermédiaire, Fouille programmée pluriannuelle 2005-2007. 
MOURRE V., THIEBAUT C. 2009 - L'industrie lithique du Moustérien final de la grotte du Noisetier (Fréchet-Aure, Hautes-Pyrénées) dans le contexte des Pyrénes centrales françaises, Treballs d'Arqueologia 14, p. 89-106.

MOURRE V., THIÉBAUT C., CHALARD P., COLONGE D., COUDENNEAU A., DESCHAMPS M., SACCO-SONADOR A. en cours - The final Mousterian on both sides of the Pyrenees. The Neanderthal Home : spatial and social behaviours, Tarragone, Abric Romani (1909-2009) International workshop (6-9 octobre 2009).

MUNOZ M., SANCHEZ-GONI F. et UGARTE F. 1989 - El entomo geo-ambiental del yacimiento arqueologico de Kurtzia. Sopela-Barrika. Costa occidental de Bizkaia. Munibe, 41, pp. 107-115.

NORMAND C. 2002 - Transition Paléolithique moyen Paléolithique supérieur et évolution de l'Aurignacien dans la salle de Saint-Martin de la grotte d'Isturitz (commune de Saint-Martin-d'Arbéroue), Opération de fouilles programmées triannuelles 2000 - 2002 Rapport final SRA d'Aquitaine.

OBERMAIER H. 1924 - Las diferentes facies del Musteriense espanol y especialmente del de los yacimientos, Revista de la Biblioteca archivo y museo, p. 143-177.

PASSEMARD E. 1936 - Le Moustérien à l'abri Olha en Pays-Basque, Revue Lorraine d'Anthropologie, $8^{\text {ème }}$ année, p. 117-160.

PASSEMARD E. 1944 - La caverne d'Isturitz en Pays Basque, Préhistoire, t. IX, fasc. un, PUF.

PEYRONY D. 1930 - Le Moustier : ses gisements, ses industries, ses couches archéologiques, Revue anthropologique, t.40, p. 48-76 et 155-176.

PLISSON H. 1986 - Analyse des polis d'utilisation sur le quartzite, In: Technical aspects of microwear studies on stone tools. Owen L.-R. et Unrath G. (dir.). Actes de la conférence de Tübingen, février 1985. Early Man News 9.10.11, p. 47-49.

RIOS J. 2007 - Industria litica y sociedad en la transicion del Paleolitico medio al Superior en torno al Golfo de Bizkaia, Universidad de Cantabria, p. 561.
ROCHE H. et TEXIER P-J. 1991 - La notion de complexité dans un ensemble lithique. Application aux séries acheuléennes d'Isenya (Kenya) , in: 25 ans d'Études technologiques en Préhistoire, Juan-les-Pins, Xlèmes Rencontres Internationales d'Archéologie et d'Histoire d'Antibes, Ed. ADPCA, p. 99-108.

SACCO-SONADOR A. 2008 - L'industrie lithique du turon de Calavanté (Hautes-Pyrénées), Université Toulouse - le Mirail. Mémoire de Master I, p.125.

SAENZ DE BURAGA A., EBRARD D. 2004 - El Musteriense de la cueva de Harregi en Aussurucq, Soule (Excavationes: Pierre Boucher, 1954-1960, Veleia, 21, p. 9-39

SLIMAK L. 2004 - Les dernières expressions du Moustérien entre Loire et Rhône, Université de Provence, Thèse de doctorat, 2 vol., $860 \mathrm{p}$.

TEXIER P.-J. 1996 - L'Acheuléen d'Isenya (Kenya), approche expérimentale des principales chaînes opératoires lithiques, La Vie Préhistorique, SPF, Ed. Faton, p. 58-63.

THIÉBAUT C. 2005 - Le Moustérien à denticulés : variabilité ou diversité techno-économique ?, Université de Provence, Thèse de Doctorat, 2 vol., $870 \mathrm{p}$.

TIXIER J. 1956 - Le hachereau dans I'Acheuléen nord-africain - Notes typologiques, Congrès préhistorique de France - Compte-rendu de la XVème session - PoitiersAngoulême - 15-22 juillet 1956, p. 914-923.

UTRILLA P. \& MAZO C. 1996 - Non-flint raw materials in La Rioja : a tentative interpretation. Non-flint stone tools and the Palaeolithic occupation of the Iberian Peninsula. N. Moloney, L. Raposo and M. Santonja, Tempus Reparatum, BAR International Series 649: 63-80.

VEGA DEL SELLA C. D. L. 1921 - El Paleolítico de Cueva Morín (Santander) y notas para la climatologia cuaternaria, Comision de Investigaciones Paleontológicas y Prehistóricas, Memoria $n^{\circ} 29$, (Serie prehistórica, $n^{\circ} 25$ ), Museo Nacional de Ciencias Naturales, 167 p. 